\title{
Bypassing UGC from some Optimal Geometric Inapproximability Results
}

\author{
Venkatesan Guruswami*
}

\author{
Prasad Raghavendra ${ }^{\dagger}$
}

Rishi Saket ${ }^{\ddagger}$
$\mathrm{Yi} \mathrm{Wu} u^{\S}$

November 15, 2010

\begin{abstract}
The Unique Games conjecture (UGC) has emerged in recent years as the starting point for several optimal inapproximability results. While for none of these results a reverse reduction to Unique Games is known, the assumption of bijective projections in the Label Cover instance seems critical in these proofs. In this work we bypass the UGC assumption in inapproximability results for two geometric problems, obtaining a tight NP-hardness result in each case.

The first problem known as the $L_{p}$ Subspace Approximation is a generalization of the classic least squares regression problem. Here, the input consists of a set of points $S=\left\{a_{1}, \ldots, a_{m}\right\} \subseteq$ $\mathbb{R}^{n}$ and a parameter $k$ (possibly depending on $n$ ). The goal is to find a subspace $H$ of $\mathbb{R}^{n}$ of dimension $k$ that minimizes the sum of the $p^{\text {th }}$ powers of the distances to the points. For $p=2, k=n-1$, this reduces to the least squares regression problem, while for $p=\infty, k=0$ it reduces to the problem of finding a ball of minimum radius enclosing all the points. We show that for any fixed $p(2<p<\infty)$ it is NP-hard to approximate this problem to within a factor of $\gamma_{p}-\epsilon$ for constant $\epsilon>0$, where $\gamma_{p}$ is the $p$ th moment of a standard Gaussian variable. This matches the factor $\gamma_{p}$ approximation algorithm obtained by Deshpande, Tulsiani and Vishnoi [12], who also showed the same hardness result under the UGC.

The second problem we study is the related $L_{p}$ Quadratic Grothendieck Maximization Problem, considered by Kindler, Naor and Schechtman [29]. Here, the input is a multilinear quadratic form $\sum_{i, j=1}^{n} a_{i j} x_{i} x_{j}$ and the goal is to maximize the quadratic form over the $\ell_{p}$ unit ball, namely all $x$ with $\sum_{i=1}^{n}\left|x_{i}\right|^{p}=1$. The problem is polytime solvable for $p=2$. We show that for any constant $p(2<p<\infty)$, it is NP-hard to approximate $\operatorname{Val}_{p}(A)$ to within a factor of $\gamma_{p}^{2}-\epsilon$ for any $\epsilon>0$. The same hardness factor was shown under the UGC in [29]. We also obtain a $\gamma_{p}^{2}$-approximation algorithm for the problem using the convex relaxation of the problem defined by [29]. A $\gamma_{p}^{2}$ approximation algorithm has also been independently obtained by Naor and Schechtman [33].

These are the first approximation thresholds, proven under $\mathrm{P} \neq \mathrm{NP}$, that involve the Gaussian random variable in a fundamental way. Note that the problem statements themselves have no mention of Gaussians.
\end{abstract}

\footnotetext{
*Computer Science Department, Carnegie Mellon University, Pittsburgh, PA 15213. guruswami@cmu.edu . Research supported in part by a Packard Fellowship.

$\dagger$ College of Computing, Georgia Institute of Technology, Atlanta, GA. praghave@cc.gatech.edu

$\ddagger$ Center for Computational Intractability, Princeton University, Princeton, NJ. rsaket@princeton.edu. Research supported in part by NSF 0832797. Some of this work was done when the author was at Carnegie Mellon University.

$\S$ IBM Almaden Research Center, San Jose, CA. wuyi@us.ibm.com. Some of this work was done when the author was at Carnegie Mellon University.
} 


\section{Contents}

1 Introduction $\quad 3$

2 Motivation and Related Work $\quad 5$

$2.1 L_{p}$ Subspace Approximation Problem $\ldots \ldots \ldots \ldots \ldots$

$2.2 L_{p}$ Quadratic Grothendieck Maximization Problem ～. . . . . . . . . . . . . . 5

2.3 Bypassing the Unique Games Conjecture . . . . . . . . . . . . . . . . . . 6

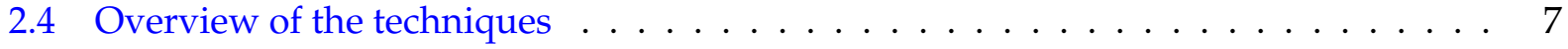

2.4 .1 NP-Hardness Reductions . . . . . . . . . . . . . . . . . 7

2.4.2 Approximating the $L_{p}$ Quadratic Grothendieck Maximization Problem . . . . 8

3 Preliminaries $r$

3.1 Smooth Label Cover . . . . . . . . . . . . . . . . . . . . . . . . . 9

3.2 Statement of results . . . . . . . . . . . . . . . . . . . . . 10

4 Hardness reduction for Subspace $(k, p) \quad 10$

4.1 Step 1: Preliminary Instance $\mathcal{A}_{\text {prel }} \ldots \ldots \ldots \ldots \ldots \ldots \ldots$

4.2 Step 2: Folding and Final Instance $\mathcal{A}_{\text {final }} \ldots \ldots \ldots \ldots \ldots \ldots \ldots \ldots$

4.3 Completeness. . . . . . . . . . . . . . . . . . . . . . . 13

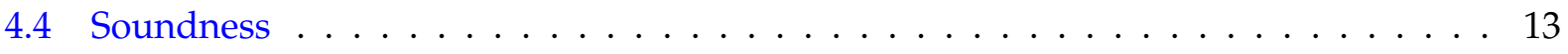

5 Hardness Reduction for $\mathrm{QM}(p) \quad 16$

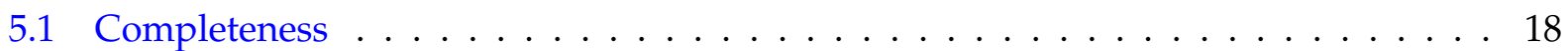

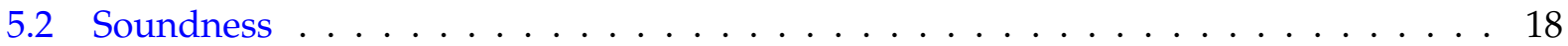

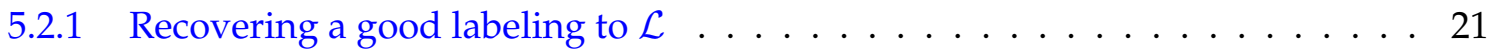

5.3 Removing the diagonal terms . . . . . . . . . . . . . . . . . 23

6 Approximation for $\mathrm{QM}(p) \quad 25$ 


\section{Introduction}

The Unique Games Conjecture of Khot [22] asserts that a certain binary constraint satisfaction problem is hard to approximate over a large enough alphabet. The conjecture has been shown to imply optimal hardness results for various important combinatorial optimization problems such as MaxCut [23], Vertex Cover [24] and more generally, constraint satisfaction problems [36]. However, arguably there has been little progress towards proving the conjecture. On the contrary, recent algorithmic results have disproved some stronger variants of the conjecture, and solved the Unique Games problem on special classes of instances like expanders [5,3]. Moreover, while the Unique Games Conjecture is known to imply optimal inapproximability results for MaxCut and Vertex Cover, the converse is unknown. In other words, this leaves open the possibility that while the implications of the conjecture are true, the conjecture itself is false. For all these reasons, it is a worthwhile endeavor to investigate if the optimal inapproximability results obtained via the Unique Games Conjecture can be shown without appealing to the conjecture. In this work, we consider two geometric problems for which optimal inapproximability results based on the Unique Games Conjecture have been shown previously, and obtain the same hardness results unconditionally, i.e., without appealing to the conjecture.

$L_{p}$ Subspace Approximation Problem. The first problem we consider is the $L_{p}$ Subspace Approximation Problem for $2 \leqslant p<\infty-$ a natural generalization of the least squares regression problem, the low rank matrix aproximation problem and the problem of computing radii of point sets. Here the input consists of a set of points $S=\left\{a_{1}, \ldots, a_{m}\right\} \subseteq \mathbb{R}^{n}$, and an integer $1 \leqslant k \leqslant n$. The goal is to find a $k$-dimensional subspace $H$ of $\mathbb{R}^{n}$ that minimizes the sum of the $p^{\text {th }}$ powers of the distances to the points in $S$. Formally, the goal is to compute:

$$
\operatorname{Sub}_{p}(S, k)=\min _{H \subseteq \mathbb{R}^{n}: \operatorname{dim}(H)=k}\left(\sum_{i=1}^{m} \operatorname{dist}\left(H, a_{i}\right)^{p}\right)^{1 / p},
$$

where dist(, ) is the usual $\ell_{2}$ distance between a subspace and a point. Informally, it is the problem of determining how close a given set of points is from lying in a smaller subspace, where the measure of closeness to a subspace is the $\ell_{p}$ norm of the tuple of Euclidean distances of the set of points from the subspace. Such problems arise naturally in classification of large data sets for applications in machine learning and data mining. As an algorithmic question, it is a generalization of various special cases for different values of $p$ such as Low rank matrix approximation $(p=2)$ or Computing the radii of point sets $(p=\infty)$. We refer the reader to [12] for a more comprehensive discussion of these connections.

In this work we focus on the hardness of approximating the $L_{p}$ Subspace Approximation Problem for the case when $k=n-1$, i.e., the problem of finding a hyperplane that is closest to the set of points in the measure defined above. Let $\gamma_{p}$ denote the $p^{\text {th }}$ moment of a normal random variable. Recently, Deshpande, Tulsiani and Vishnoi [12] obtained a $\gamma_{p}$ approximation for the problem, and showed a matching hardness assuming the Unique Games Conjecture. Bypassing the need for the UGC, we obtain a $\gamma_{p}$ hardness of approximation unconditionally.

Theorem. For any given $p(2<p<\infty)$ the $L_{p}$ Subspace Approximation Problem is NP-hard to approximate within a factor of $(1-\epsilon) \gamma_{p}$ for any $\epsilon>0$. 
$L_{p}$ Grothendieck Problem. The second problem we consider is that of maximizing a multilinear quadratic form over the unit $\ell_{p}$ ball in $\mathbb{R}^{n}$ for constant $p, 2 \leqslant p<\infty$. Formally, the input to the problem is a symmetric $n \times n$ matrix $A=\left(a_{i j}\right)$ with zero diagonal entries, the goal is to compute the following quantity,

$$
\operatorname{Val}_{p}(A):=\max \left\{\left.\sum_{i, j=1}^{n} a_{i j} x_{i} x_{j}\left|\sum_{i}\right| x_{i}\right|^{p} \leqslant 1\right\},
$$

We shall refer to this problem as the $L_{p}$ Quadratic Grothendieck Maximization Problem. In the case where $p=2, \operatorname{Val}_{2}(A)$ is nothing but the maximum eigenvalue of the matrix $A$ and hence is computationally tractable. The case $p=\infty$ is commonly referred to as the Grothendieck problem and has been extensively studied in mathematics and computer science for its applications to combinatorial optimization, graph theory and correlation clustering $[34,2,1,11]$. The case when $2<p<\infty$ has applications towards studying spin glass systems in physics (See Section 2.2)

Kinder, Naor and Schectman [29] obtained a $\left(\gamma_{p}^{2}-\epsilon\right)$-hardness for every $\epsilon$ for the $L_{p}$-Grothendieck problem assuming the UGC, and also exhibited an almost matching $\frac{p}{e}+30 \log p$-approximation algorithm. Bypassing the Unique Games Conjecture, we obtain a $\gamma_{p}^{2}$ hardness unconditionally for the problem.

Theorem. For any constant $p>2$, it is NP-hard to approximate $\operatorname{Val}_{p}(A)$ to within $\gamma_{p}^{2}-\epsilon$ for any $\epsilon>0$.

Furthermore, we also obtain an approximation algorithm that exactly matches the above hardness result for every $p$.

Theorem. There is a polynomial time algorithm to approximate $\operatorname{Val}_{p}(A)$ to within $\gamma_{p}^{2}$ for any symmetric matrix $A$ with all diagonal entries equal to zero.

A $\gamma_{p}^{2}$ approximation for $\operatorname{Val}_{p}(A)$ has also been independently obtained by Naor and Schechtman [33] as part of a more general result.

The above mentioned NP-hardness results are noteworthy for a couple of reasons. First, the inapproximability factors for both the problems are irrational numbers arising from the Gaussian distribution, although neither of the problems involve the Gaussian distribution directly. Inapproximability factors arising from properties of the Gaussian distribution have previously been obtained for other problems - such as Maximum Cut [23] — using the Unique Games Conjecture, reductions based on which naturally involve the Gaussian distribution via analytic tools such as the Invariance Principle [32].

Second, the inapproximability factors obtained in each case arise directly from a semidefinite program for the problem. Again, the optimality of semidefinite programs has been a recurring theme in UGC hardness results, while this result is among the few NP-hardness results that highlight this phenomenon.

Third, the reductions in this work are based on a dictatorship test which quantitatively utilizes the Central Limit Theorem, i.e. the distribution of a sum of significant number of independent Bernoulli random variables is close to a Gaussian distribution. This is precisely the reason for the appearance of the Gaussian distribution. A key ingredient in our reductions is the smooth version of Label Cover which enables us to devise a more sophisticated decoding procedure which can be 
combined with the dictatorship test. It is pertinent to note that a couple of the (few) previous results using smooth versions of Label Cover, on hardness of learning intersection of halfspaces [26] and monomials [16], have also used analysis based on versions of the Central Limit Theorem. This work suggests the possibility of obtaining optimal NP-hardness results for other combinatorial optimization problems using reductions based on smooth versions of Label Cover.

\section{Motivation and Related Work}

\section{1 $L_{p}$ Subspace Approximation Problem}

Algorithmically various special cases of this problem have been well studied. For $p=2$ it reduces to the problem of determining a rank $k$ approximation $B$ to an $n \times m$ matrix $A$ with respect to the Frobenius norm, which can be computed in polynomial time by using Singular Value Decomposition of $A$ [17]. Efficient $(1+\epsilon)$ approximations have been given various cases such as: for $p=1$ and constant $k$ by Feldman $e t$ al. [15]; $p=\infty$ and constant $k$ by Har-Peled and Varadarajan [20]; and for general $p$ and constant $k$ by Shyamalkumar and Varadarajan [38] and Deshpande and Varadarajan [13]. On the other hand, the problem can be approximated to within $O(\sqrt{\log m})$ for any value of $k$ for $p=\infty$ as shown by Varadarajan et al. [40] building on the work of Nemirovski et al. [34].

On the complexity front, Brieden, Gritzman and Klee [9] showed that the problem is NP-hard to solve optimally for $k=n-1$ and $p=\infty$. Subsequently, the problem was shown to be NP-hard to approximate within $(\log m)^{\delta}$ for $k \leqslant n-n^{\epsilon}$ for any $0<\epsilon<1$ and $p=\infty$ [40].

In more recent work, Deshpande,Tulsiani and Vishnoi [12] gave a $\sqrt{2} \gamma_{p}$ approximation for this problem for any $k$ and any $p \geqslant 2$, and a $\gamma_{p}$ approximation factor when $k=n-1$. Assuming the Unique Games Conjecture they also prove that the problem is hard approximate within a factor of $(1-\epsilon) \gamma_{p}$.

\section{2 $L_{p}$ Quadratic Grothendieck Maximization Problem}

The special case of the problem when $p=\infty$ (maximizing over the hypercube), has been extensively studied. The problem is known to admit an $O(\log n)$ approximation $[35,34,31,11]$. On the other hand, it was shown to be NP-hard to approximate within some constant factor in [2] and [11]. In [4], Arora et al. gave the best known inapproximability factor of $(\log n)^{c}$ for some $c>0$ for this problem.

The $L_{p}$ Quadratic Grothendieck Maximization Problem for constant $p$ such that $2<p<\infty$ has received attention more recently in the work of Kindler, Naor and Schechtman [29]. They exhibit an algorithm to approximate $\operatorname{Val}_{p}(A)$ to within a factor of $\frac{p}{e}+30 \log p$ and also show a Unique Games Conjecture [22] based inapproximability factor of $\gamma_{p}^{2}-\epsilon$ for all $\epsilon>0$. Here $\gamma_{p}$ denotes the $p$ th moment of a standard Gaussian variable. Note that while asymptotically (i.e. as $p \rightarrow \infty)$ the upper and lower bounds both tend to $\frac{p}{e}(1+o(1))$, for a fixed constant $p(2<p<\infty)$, there remained a gap between them.

The $L_{p}$ Grothendieck problem for $2<p<\infty$ has application towards computing the ground 
states of spin glasses in a hard potential well. In the spin glass physical model, the spin of particles in certain physical systems are represented by variables $x_{i}$ for the $i$ th particle. Any pair of particles, say $i$ and $j$, have an energy proportional to their product i.e. $x_{i} x_{j}$. Given a symmetric matrix $A=\left(a_{i j}\right)$ of pairwise coefficients the total energy of the system is $\sum_{i, j=1}^{n} a_{i j} x_{i} x_{j}$. The system is additionally constrained by external factors to force the vector $\left(x_{1}, \ldots, x_{n}\right)$ to be within (say) a convex body. Thus the problem of minimizing the total energy of the system boils down to computing $\operatorname{Val}_{p}(-A)$ if the convex body is the $\ell_{p}$ unit ball. For more details on the connections to physics we refer the reader to [29].

More generally, the problem of maximizing a multilinear quadratic form over a given convex set of $\mathbb{R}^{n}$ has numerous applications. For instance, the special case when the convex set is a polytope, lies at the core of non-linear optimization and has been studied for its applications in operations research and economics [8]. The special case of the problem where the convex set is a simplex arises in computational biology for analyzing genomic frequencies from incomplete data [19].

\subsection{Bypassing the Unique Games Conjecture}

The Unique Games Conjecture of Khot [22] states that a special type of two variable constraint satisfaction problem over a large enough label set - which we shall refer to as an instance of Unique Games - is hard to approximate within any constant factor, even when almost all of the constraints are satisfiable. Each of the two variable constraints of Unique Games is such that for every assignment of a label to one variables, there is a unique label that can be assigned to the other variable to satisfy the constraint. The conjecture has been used to prove several hardness of approximation results, many of them optimal, for a large class of CSPs [36] including Maximum Cut [23], covering problems such as Vertex Cover [24] and scheduling problems [6, 7] among others. It has also inspired unconditional results in integrality gap constructions and lower bounds in metric embedding, most notably in the work of Khot and Vishnoi [28] and subsequent related works [14, 27, 37]. On the algorithmic side several approximation algorithms have been given for Unique Games [22, 39, 18], with the ones given by Charikar, Makarychev and Makarychev [10] being optimal in the sense that improving them slightly would disprove the conjecture. Subsequent work by Arora et al. [5] showed that Unique Games is tractable when the constraint graph is an expander.

The above algorithms were based on rounding the solution of linear programming (LP) or semi-definite programming (SDP) relaxations. In more recent work Kolla [30] gave an approximation algorithm for Unique Games based purely on spectral techniques. Using a similar approach, Arora, Barak, and Steurer [3] have recently obtained a $2^{O\left(n^{\epsilon}\right)}$ time constant factor approximation for Unique Games instances in which $1-\epsilon$ fraction of constraints are satisfiable. While the algorithm is not polynomial time, it does imply that for the conjecture to be true, any polynomial time reduction from 3-SAT to Unique Games must increase the instance size substantially, unless 3-SAT has subexponential time algorithms.

On the complexity side however, there has been little progress in proving the Unique Games Conjecture. In light of this lack of progress in proving the conjecture, we believe that it is a worthwhile goal to investigate whether various hardness results based on Unique Games Conjecture do indeed require its assumption. 


\subsection{Overview of the techniques}

In the next few paragraphs we give an informal description of the techniques used in proving the results of the paper and the new ingredients employed to build upon the work of [29] and [12].

\subsubsection{NP-Hardness Reductions}

Our hardness of approximation results for both the $L_{p}$ Quadratic Grothendieck Maximization Problem and the $L_{p}$ Subspace Approximation Problem are via reductions from an instance of Smooth Label Cover which is two variable CSP and a variant of the commonly known Label Cover problem. It was first introduced in [21] for proving hardness results in hypergraph coloring and subsequently utilized for other applications in [25] [26] and [16].

For the $L_{p}$ Quadratic Grothendieck Problem our overall approach is similar to that of [29]: use the quadratic form to simulate a long code test on the vertices of the Smooth Label Cover (or Unique Games in [29]). As before, the coordinates are the union of long codes for each vertex of Smooth Label Cover instance. The quadratic form is given in terms of the Fourier coefficients of the various long codes. In our case however, the quadratic form differs from that of [29] in order to avoid the usage of the Cauchy-Schwarz inequality in analyzing the long code test as it requires the uniqueness property of the constraints afforded by an instance of Unique Games. More specifically, the quadratic form in our construction simulates the so-called dictatorship and consistency tests on the long codes. The dictatorship test yields a small set each of influential labels for a significant fraction of vertices. This is combined with the consistency test to obtain a good labeling to the instance of Smooth Label Cover. Our analysis crucially depends on the smoothness property of the constraints of the instance which roughly stated is: for any vertex, given a small set of labels, most of the constraints involving the vertex restricted to that set of labels appear structurally similar to constraints of an instance of Unique Games. In some sense the constraints incident on any given vertex of the Smooth Label Cover are locally unique for any given small set of labels. This enables us to design a two step decoding procedure which extracts the aforementioned good labeling to the instance.

The reduction for the $L_{p}$ Subspace Approximation Problem also follows a similar approach. Analogous to the construction of [12], there is a coordinate for every label of every vertex of the Smooth Label Cover instance. The point sets simulate the dictatorship test on the vertices. However, unlike in [12] we utilize the method of folding to automatically ensure consistency. This involves a change of basis and subsequently projecting the instance on a smaller subspace which depends on the constraints of the instance. This is not applicable to the Unique Games Conjecture based reduction of [12] as it lacks perfect completeness. This method of folding is also not applicable to the reduction for the $L_{p}$ Quadratic Grothendieck Maximization Problem since in that case the $\ell_{p}$ norms are not preserved under basis transformations. The rest of the analysis including the two step decoding procedure to obtain a good labeling to the instance of Smooth Label Cover is similar to that for the $L_{p}$ Quadratic Grothendieck Maximization Problem. 


\subsubsection{Approximating the $L_{p}$ Quadratic Grothendieck Maximization Problem}

Our algorithm is essentially a simplification of the techniques in [29]. We define the following convex relaxation for $\operatorname{Val}_{p}(A)$ :

$$
\operatorname{Vec}_{p}(A)=\max \left\{\sum_{i, j=1}^{n} a_{i j}\left\langle v_{i}, v_{j}\right\rangle:\left\{v_{1}, \ldots, v_{n}\right\} \subseteq L_{2}, \sum_{i=1}^{n}\left\|v_{i}\right\|_{2}^{p} \leqslant 1\right\} .
$$

As observed in [29] the above convex program can be solved in polynomial time to arbitrary small precision. We directly show that $\operatorname{Vec}_{p}(A)$ is a $\gamma_{p}^{2}$ approximation to $\operatorname{Val}_{p}(A)$. This can be easily derived from the following fact: there exist mean zero Gaussian random variables $h_{i}$ for $i=1, \ldots, n$ such that $\mathbb{E}\left[h_{i} h_{j}\right]=\left\langle v_{i}, v_{j}\right\rangle$. Writing $\operatorname{Vec}_{p}(A)$ as $\mathbb{E}\left[\sum_{i, j=1}^{n} a_{i j} h_{i} h_{j}\right]$ and normalizing each variable by $\left(\sum_{k=1}^{n}\left|h_{k}\right|^{p}\right)^{1 / p}$ yields the desired approximation.

A simple proof with the details is given in Section 6. This differs from the proof of [29] which obtains a slightly weaker approximation via a truncation based rounding algorithm. A generalization of our approximation for convex bodies has been obtained independently by Naor and Schechtman [33] and for the unit $\ell_{p}(p>2)$ ball it essentially gives the same result as ours. Our proof also yields a polynomial time rounding algorithm to compute a solution $\left\{x_{i}\right\}_{i=1}^{n}$ which approximates $\operatorname{Val}_{p}(A)$ to within a factor of $\gamma_{p}^{2}(1+\delta)$ for arbitrarily small $\delta>0$.

\section{Preliminaries}

We begin this section by first formally defining the two problems that we study.

Definition 3.1. The $L_{p}$ Subspace Approximation Problem, which we denote by Subspace $(k, p)$ where $k$ is a parameter (possibly depending on $n$ ) is: given a set of points $S=\left\{a_{1}, \ldots, a_{m}\right\} \subseteq \mathbb{R}^{n}$, to compute the following quantity,

$$
\operatorname{Sub}_{p}(S, k)=\min _{H \subseteq \mathbb{R}^{n}: \operatorname{dim}(H)=k}\left(\sum_{i=1}^{m} \operatorname{dist}\left(H, a_{i}\right)^{p}\right)^{1 / p},
$$

where the minimum is taken over all $k$-dimensional subspaces of $\mathbb{R}^{n}$ and $\operatorname{dist}(H, a)$ is the minimum Euclidean distance between $a$ and any point in $H$.

Definition 3.2. The $L_{p}$ Quadratic Grothendieck Maximization Problem which we denote as $\mathrm{QM}(p)$ for $1 \leqslant p<\infty$ is: given a symmetric matrix $A \in \mathbb{R}^{n \times n}$ with diagonal entries all zero, to compute the following quantity,

$$
\operatorname{Val}_{p}(A):=\max \left\{\left.\sum_{i, j=1}^{n} a_{i j} x_{i} x_{j}\left|\sum_{i=1}^{n}\right| x_{i}\right|^{p} \leqslant 1\right\} .
$$

We shall denote by $\gamma_{p}$ the $p$ th moment of a standard Gaussian random variable. Formally,for any $p \geqslant 0$,

$$
\gamma_{p}:=\left(\mathbb{E}\left[|g|^{p}\right]\right)^{1 / p}
$$

where $g$ is a Gaussian random variable with mean 0 and variance 1 . 
The analysis of the dictatorship tests in our reductions, requires lower bounds on the moments of sums of independent Bernoulli variables. The following lemma, proved in [29] (as Lemma 2.5) gives us the required bound.

Lemma 3.3. Let $X_{1}, \ldots, X_{n}$ be independent Bernoulli random variables such that $\mathbb{E}\left[X_{i}\right]=0$ for all $1 \leqslant$ $i \leqslant n$ and $\sum_{j=1}^{n} \mathbb{E}\left[X_{j}^{2}\right]=1$. Assume that for some $\tau \in\left(0, e^{-4}\right)$, we have $\sum_{j=1}^{n} \mathbb{E}\left[\left|X_{j}\right|^{3}\right] \leqslant \tau$. Then for every $p \geqslant 1$,

$$
\left(\mathbb{E}\left[\left|\sum_{j=1}^{n} X_{j}\right|^{p}\right]\right)^{1 / p} \geqslant \gamma_{p} \cdot\left(1-4 \tau(\log (1 / \tau))^{p / 2}\right) .
$$

\subsection{Smooth Label Cover}

Our reductions require a special variant of the usual Label Cover problem, which is formally defined as follows.

Definition 3.4. An instance of Smooth Label Cover $\mathcal{L}\left(G(V, E), N, M,\left\{\pi^{v, e} \mid e \in E, v \in e\right\}\right)$ consists of a regular connected (undirected) graph $G(V, E)$ with vertex set $V$ and edge set E. Every edge $e=\left(v_{1}, v_{2}\right)$ is associated with projection functions $\left\{\pi^{e, v_{i}}\right\}_{i=1}^{2}$ where $\pi^{e, v_{i}}:[M] \rightarrow[N]$. A vertex labeling is a mapping defined on $L: V \rightarrow[M]$. A labeling $L$ satisfies edge $e=\left(v_{1}, v_{2}\right)$ if $\left.\pi^{e, v_{1}}\left(L\left(v_{i}\right)\right)=\pi^{e, v_{2}}\left(L\left(v_{j}\right)\right)\right)$. The goal is to find a labeling which satisfies the maximum number of edges.

The following theorem states the hardness of approximation for the Smooth Label Cover problem and also describes the various structural properties, including smoothness, that are satisfied by the hard instances. The proof of the theorem appears in [16] and we omit it.

Theorem 3.5. There exists a constant $c_{0}>0$ such that for any constant integer parameters $J, u \geqslant$ 1 , it is NP-hard to distinguish between the following two cases for a Smooth Label Cover instance $\mathcal{L}\left(G(V, E), N, M,\left\{\pi^{v, e} \mid e \in E, v \in e\right\}\right)$ with $M=7^{(J+1) u}$ and $N=2^{u} 7^{J}$ :

- (YES Case). There is a labeling that satisfies every edge.

- (NO Case). There is no labeling that satisfies $2^{-c_{0} u}$ fraction of the edges.

In addition, the instance $\mathcal{L}$ satisfies the following properties:

- (Smoothness) For any vertex $w \in V$,

$$
\forall i, j \in[M], \operatorname{Pr}\left[\pi^{w, e}(i)=\pi^{w, e}(j)\right] \leqslant 1 / J,
$$

where the probability is over a randomly chosen edge incident on $w$.

- The degree of the (regular) graph $G$, which we denote by $d$ is a constant depending only on $u$ and J.

- For any vertex $v$, edge e incident on $v$, and any element $i \in[N]$, we have $\left|\left(\pi^{e, v}\right)^{-1}(i)\right| \leqslant t:=4^{u}$; i.e., there are at most $t=4^{u}$ elements in $[M]$ that are mapped to the same element in $[N]$.

- (Weak Expansion)For any $\delta>0$, let $V^{\prime} \subseteq V$ and $\left|V^{\prime}\right|=\delta \cdot|V|$, then the number of edges among the vertices in $\left|V^{\prime}\right|$ is at least $\left(\delta^{2} / 2\right)|E|$. 


\subsection{Statement of results}

We conclude this section by formally stating as theorems the results we prove in the following sections.

Theorem 3.6. For any fixed $p>2$ and constant $\epsilon>0$, there is a polynomial time reduction from an instance $\mathcal{L}$ of Smooth Label Cover with appropriately chosen parameters $J$ and $u$ to an instance $A$ of $\mathrm{QM}(p)$ such that,

- (Completeness) If $\mathcal{L}$ is a YES instance, then $\operatorname{Val}_{p}(A)=1$.

- (Soundness) If $\mathcal{L}$ is a NO instance, then $\operatorname{Val}_{p}(A) \leqslant \gamma_{p}^{-2}(1+\epsilon)$.

The above implies that it is NP-hard to approximate $\mathrm{QM}(p)$ within a factor of $(1-\epsilon) \gamma_{p}^{2}$ for all $\epsilon>0$.

The above theorem is proved in Section 5. The following theorem, proved in Section 6, shows that Theorem 3.6 is essentially tight.

Theorem 3.7. For any fixed $p \geqslant 2, \operatorname{Vec}_{p}(A) \leqslant \gamma_{p}^{2} \cdot \operatorname{Val}_{p}(A)$ for any instance $A$ of $\mathrm{QM}(p)$. This implies a polynomial time $\gamma_{p}^{2}$ approximation for $\mathrm{QM}(p)$.

Furthermore, there is a polynomial time (randomized) rounding procedure that rounds the solution to $\operatorname{Vec}_{p}(A)$ to obtain $a(1+\epsilon) \gamma_{p}^{2}$ approximate solution to $\operatorname{Val}_{p}(A)$.

Theorems 3.6 and 3.7 together give the results for the $L_{p}$ Quadratic Grothendieck Maximization Problem obtained in this paper.

For the $L_{p}$ Subspace Approximation Problem, the following theorem proved in Section 4 yields the desired hardness of approximation.

Theorem 3.8. For any fixed $p>2$ and $\epsilon>0$, there is polynomial time reduction from an instance $\mathcal{L}$ of Smooth Label Cover with appropriately chosen parameters $J$ and $u$ to a set of points $S \subseteq \mathbb{R}^{n}$ as an instance of Subspace $(n-1, p)$ such that,

- (Completeness) If $\mathcal{L}$ is a YES instance, then $\operatorname{Sub}_{p}(n-1, S)=1$.

- (Soundness) If $\mathcal{L}$ is a NO instance, then $\operatorname{Sub}_{p}(n-1, S) \geqslant \gamma_{p}(1-\epsilon)$.

The above implies that it is NP-hard to approximate $\operatorname{Subspace}(n-1, p)$ within a factor of $(1-\epsilon) \gamma_{p}$ for all $\epsilon>0$.

Note on notation. In the following sections, the parameter $n$ need not denote the size of the instances of $\mathrm{QM}(p)$ or Subspace $(k, p)$ and its definition shall be made clear at the beginning of each section.

\section{Hardness reduction for Subspace $(k, p)$}

In this section we shall describe the NP-hardness reduction from Smooth Label Cover to the $L_{p}$ Subspace Approximation Problem for a fixed $p>2$. Let the Smooth Label Cover instance be 
$\mathcal{L}\left(G(V, E), N, M,\left\{\pi^{v, e} \mid e \in E, v \in e\right\}\right)$. We shall choose the parameters $J$ and $u$ as part of the analysis in Section 4.4. For convenience let $n:=|V|$. Note that $n$ does not correspond to the dimension of the point set constructed in the reduction. We shall not explicitly calculate the dimension, but shall use the notation dim to denote it. The set of points constructed shall be an instance of Subspace $(\operatorname{dim}-1, p)$. The Euclidean distance of a point from a dimension $(\operatorname{dim}-1)$ subspace, or a hyperplane through origin, is same as the magnitude of the dot product of that point with the unit normal vector. Therefore the problem Subspace $(\operatorname{dim}-1, p)$ is same as computing the unit normal vector which minimizes the sum of the $p$ th powers of the dot products of the given points with the vector. Our reduction will follow this formulation, with the goal being to compute such a unit normal vector. The reduction proceeds in two steps: the first step yields a preliminary instance consisting of a set of points and the second step applies a folding operation to generate the final instance.

For notational convenience, in this section vectors shall be represented as boldface characters.

\subsection{Step 1: Preliminary Instance $\mathcal{A}_{\text {prel }}$}

We begin by constructing the set of coordinates over which the instance is defined. For any vertex $v \in V$, let $\mathcal{M}_{v}$ be the set of coordinates $\{(v, i) \mid i \in[M]\}$, and $\mathcal{M}=\cup_{v \in V} \mathcal{M}_{v}$. In other words, $\mathcal{M}$ contains a coordinate for every label of every vertex. The instance $\mathcal{A}_{\text {prel }}$ shall be over the space $\mathbb{R}^{\mathcal{M}}$ consisting of points constructed as follows.

For every vertex $v \in V$, let the set $X^{v}$ be the set of all points in $\mathbb{R}^{\mathcal{M}}$ which are zero in the coordinates not corresponding to $v$ i.e. $\mathcal{M} \backslash \mathcal{M}_{v}$ and take the values $\{-1,1\}$ in the $M$ coordinates $\mathcal{M}_{v}$ corresponding to $v$. More formally,

$$
X^{v}:=\left\{\mathbf{x} \in \mathbb{R}^{\mathcal{M}} \mid \forall i \in[M], \mathbf{x}\left(v^{\prime}, i\right) \in\{-1,1\} \text { if } v^{\prime}=v \text { and } 0 \text { otherwise }\right\} .
$$

The instance $\mathcal{A}_{\text {prel }}$ consists of the point set $X:=\cup_{v \in V} X^{v}$.

Consider a vector $\mathbf{b} \in \mathbb{R}^{\mathcal{M}}$. For any vertex $v \in V$, define $\mathbf{b}_{v}$ to be the vector which is same as $\mathbf{b}$ in the $M$ coordinates $\mathcal{M}_{v}$ and zero in rest of the coordinates. Define the function $f_{\mathbf{b}_{v}}$ over $\{-1,1\}^{\mathcal{M}_{v}}$ as $f_{\mathbf{b}_{v}}(\mathbf{x}):=\left\langle\mathbf{b}_{v}, \mathbf{x}\right\rangle$ for all $\mathbf{x} \in\{-1,1\}^{\mathcal{M}_{v}}$. Define the $q$-norm $\left\|f_{\mathbf{b}_{v}}\right\|_{q}:=$ $\left(\mathbb{E}_{\mathbf{x} \in\{-1,1\}^{\mathcal{M}_{v}}}\left[\left|f_{\mathbf{b}_{v}}(\mathbf{x})\right|^{q}\right]\right)^{1 / q}$ for all $q \geqslant 1$. It is easy to see that $\left\|f_{\mathbf{b}_{v}}\right\|_{2}^{2}=\left\|\mathbf{b}_{v}\right\|_{2}^{2}:=\sum_{j=1}^{M} \mathbf{b}(v, i)^{2}$.

Using the above definitions, given $\mathcal{A}_{\text {prel }}$ as an instance, the problem of Subspace $(\operatorname{dim}-1, k)$ is equivalent to computing a unit normal vector $\mathbf{b}$ that minimizes $\mathbb{E}_{v \in V}\left[\left\|f_{\mathbf{b}_{v}}\right\|_{p}^{p}\right]$. More formally, $\mathcal{A}_{\text {prel }}$ as an instance of $\operatorname{Subspace}(\operatorname{dim}-1, k)$ is equivalent to the following optimization problem:

$$
\begin{array}{lc} 
& \min \left(\mathbb{E}_{v \in V}\left[\left\|f_{\mathbf{b}_{v}}\right\|_{p}^{p}\right]\right)^{1 / p} \\
\text { subject to } & \mathbb{E}_{v \in V}\left[\left\|\mathbf{b}_{v}\right\|_{2}^{2}\right]=1
\end{array}
$$

In the next step we shall use folding to implicitly induce additional constraints on the structure of the vector $\mathbf{b}$, which incorporates the projection constraints of the edges and enables a "good" solution $\mathbf{b}$ to be decoded into a "good" labeling of the Smooth Label Cover instance $\mathcal{L}$. 


\subsection{Step 2: Folding and Final Instance $\mathcal{A}_{\text {final }}$}

For any edge $e=(u, v)$ and element $j \in[N]$, define the vector $\mathbf{h}_{j}^{e}$ as follows,

$$
\mathbf{h}_{j}^{e}(w, i)= \begin{cases}1 & \text { if } w=u \text { and } i \in\left(\pi^{e, u}\right)^{-1}(j) \\ -1 & \text { if } w=u \text { and } i \in\left(\pi^{e, v}\right)^{-1}(j) \\ 0 & \text { otherwise. }\end{cases}
$$

The above implies that for any vector $\mathbf{b} \in \mathbb{R}^{\mathcal{M}}$,

$$
\forall e=(u, v) \in E, j \in[N], \quad \mathbf{b} \perp \mathbf{h}_{j}^{e} \Leftrightarrow \sum_{i \in\left(\pi^{e, u}\right)^{-1}(j)} \mathbf{b}(u, i)=\sum_{i^{\prime} \in\left(\pi^{e, v}\right)^{-1}(j)} \mathbf{b}\left(v, i^{\prime}\right)
$$

We now define the subspace $H$ of $\mathbb{R}^{\mathcal{M}}$ as,

$$
H:=\operatorname{span}\left(\left\{\mathbf{h}_{j}^{e} \mid e \in E, j \in[N]\right\}\right) .
$$

Let $\mathbb{R}^{\mathcal{M}}=F \oplus H$ where $F \perp H$ is a subspace of $\mathbb{R}^{\mathcal{M}}$. The point set $X$ constructed in Step 1 is folded over $H$, i.e. each point in $X$ is replaced (with multiplicity) with its orthogonal projection on $F$. Let the resultant set of points be $\bar{X}$, which constitutes the final instance $\mathcal{A}_{\text {final }}$ which is in the real space $F$. The point set can be written in some orthonormal basis for $F$ and the expected solution, say $\mathbf{b}$ is also expected to lie in $F$.

Let $\overline{\mathbf{x}} \in \bar{X}$ be the orthogonal projection of a point $x \in X$ onto the subspace $F$, and let $\mathbf{b} \in F$ be any vector. Clearly we have,

$$
\langle\mathbf{b}, \overline{\mathbf{x}}\rangle=\langle\mathbf{b}, \mathbf{x}\rangle .
$$

Also, since $\mathbf{b} \perp H$, we have from Equation 6,

$$
\forall e=(u, v) \in E, j \in[N], \sum_{i \in\left(\pi^{e, u}\right)^{-1}(j)} \mathbf{b}(u, i)=\sum_{i^{\prime} \in\left(\pi^{e, v}\right)^{-1}(j)} \mathbf{b}\left(v, i^{\prime}\right) .
$$

We note that the objective value, which can be written as $\mathbb{E}_{\mathbf{x} \in X}\left[|\langle\mathbf{b}, \mathbf{x}\rangle|^{p}\right]$, is unchanged under transformation of orthonormal basis since it is a function of inner product of vectors. Similarly, the condition that $\mathbb{E}_{v}\left[\left\|\mathbf{b}_{v}\right\|_{2}^{2}\right]=\mathbb{E}_{v}\left[\left\|f_{\mathbf{b}_{v}}\right\|_{2}^{2}\right]=1$ is unchanged as well. Therefore, the folding operation only ensures that the constraints given by Equation 8 are satisfied. Therefore, the instance $\mathcal{A}_{\text {final }}$ of Subspace $(\operatorname{dim}-1, p)$ is equivalent to the following optimization problem over solutions $\mathbf{b} \in \mathbb{R}^{\mathcal{M}}$ :

subject to ,

$$
\min \left(\mathbb{E}_{v \in V}\left[\left\|f_{\mathbf{b}_{v}}\right\|_{p}^{p}\right]\right)^{1 / p}
$$

$$
\begin{gathered}
\mathbb{E}_{v \in V}\left[\left\|\mathbf{b}_{v}\right\|_{2}^{2}\right]=1, \\
\text { and } \forall e=(u, v) \in E, j \in[N], \\
\sum_{i \in\left(\pi^{e, u}\right)^{-1}(j)} \mathbf{b}(u, i)=\sum_{i^{\prime} \in\left(\pi^{e, v}\right)^{-1}(j)} \mathbf{b}\left(v, i^{\prime}\right) .
\end{gathered}
$$

Note that the last condition is equivalent to $\mathbf{b} \perp H$. 


\subsection{Completeness.}

If the instance $\mathcal{L}$ of Smooth Label Cover is a YES instance then there is a labeling $L$ of the vertices of $\mathcal{L}$ that satisfies all the edges. Using this we shall construct a solution $\mathbf{b}^{*}$ to the instance $\mathcal{A}_{\text {final }}$ as follows: for any vertex $v \in V$ and element $i \in M, \mathbf{b}^{*}(v, i)$ is 1 if $L(v)=i$ and 0 otherwise.

Since $L$ satisfies all edges, $\pi^{e, u}(L(u))=\pi^{e, v}(L(v))$ for all edges $e=(u, v)$. Therefore it is easy to see that $\mathbf{b} \perp H$. Moreover, since there is exactly one nonzero coordinate corresponding to each vertex on which $\mathbf{b}^{*}$ is 1 , we have

$$
\left\|\mathbf{b}_{v}^{*}\right\|_{2}=\left\|f_{\mathbf{b}_{v}^{*}}\right\|_{p}=1,
$$

for all $v \in V$. Therefore, $\mathbf{b}^{*}$ is a valid solution for $\mathcal{A}_{\text {final }}$ with objective value 1 .

\subsection{Soundness}

For a contradiction assume that $\mathbf{b} \in \mathbb{R}^{\mathcal{M}}$ is a solution to the instance $\mathcal{A}_{\text {final }}$ such that,

$$
\mathbb{E}_{v \in V}\left[\left\|f_{\mathbf{b}_{v}}\right\|_{p}^{p}\right] \leqslant \gamma_{p}^{p}(1-\eta),
$$

where $\eta>0$ is a positive constant. We begin with a lemma upper bounding the $\ell_{2}^{2}$ mass of blocks of coordinates in $\mathbf{b}$ corresponding to small sets of vertices.

Lemma 4.1. Let $S \subseteq V$ be a set of size $\theta|V|=\theta$ for some $0<\theta<1$. Then,

$$
\sum_{v \in S}\left\|\mathbf{b}_{v}\right\|_{2}^{2} \leqslant \gamma_{p}^{2} \theta^{1-2 / p} n
$$

Proof. We need to upper bound $\beta$ where,

$$
\sum_{v \in S}\left\|\mathbf{b}_{v}\right\|_{2}^{2}=\beta n
$$

Note that the above implies that,

$$
\mathbb{E}_{v \in S}\left[\left\|\mathbf{b}_{v}\right\|_{2}^{2}\right]=\frac{\beta}{\theta}
$$

We know from our assumption that $\mathbb{E}_{v \in V}\left[\left\|f_{\mathbf{b}_{v}}\right\|_{p}^{p}\right] \leqslant \gamma_{p}^{p}$. This implies,

$$
\begin{array}{rlll}
\gamma_{p}^{p} & \geqslant \quad \mathbb{E}_{v \in V}\left[\left\|f_{\mathbf{b}_{v}}\right\|_{p}^{p}\right] & \\
& \geqslant \quad \mathbb{E}_{v \in V}\left[\left\|f_{\mathbf{b}_{v}}\right\|_{2}^{p}\right] & & \left(\text { since }\|f\|_{p} \geqslant\|f\|_{2}\right) \\
& \geqslant \quad \mathbb{E}_{v \in V}\left[\left\|\mathbf{b}_{v}\right\|_{2}^{p}\right] & & \left(\text { since }\left\|f_{\mathbf{b}_{v}}\right\|_{2}=\left\|\mathbf{b}_{v}\right\|_{2}\right) \\
& \geqslant \quad \theta \mathbb{E}_{v \in S}\left[\left\|\mathbf{b}_{v}\right\|_{2}^{p}\right] & & \text { (by averaging) } \\
& \geqslant \theta\left(\mathbb{E}_{v \in S}\left[\left\|\mathbf{b}_{v}\right\|_{2}^{2}\right]\right)^{\frac{p}{2}} & & \text { (by Jensen's Inequality) } \\
& =\quad \theta\left(\frac{\beta}{\theta}\right)^{\frac{p}{2}} & & \text { (by Equation 14). }
\end{array}
$$

Therefore, $\beta \leqslant \gamma_{p}^{2} \theta^{1-2 / p}$ which completes the proof of the lemma. 
We next introduce the notion, similar to the one used in Section 5.2, of an irregular vertex : $v$ is said to be irregular if there is a coordinate $(v, i)$ for some $i \in[M]$ such that the value $|\mathbf{b}(v, i)|$ is large as compared to $\left\|\mathbf{b}_{v}\right\|_{2}$. Formally we have the following definition.

Definition 4.2. ( $\tau$-irregular vertex) $A$ vertex $v \in V$ is said to be $\tau$-irregular if there exists $i \in[M]$ such that $|\mathbf{b}(v, i)|>\tau\left\|\mathbf{b}_{v}\right\|_{2}$. If not, the vertex is referred to as $\tau$-regular.

The following lemma follows from Lemma 2.5 of [29] in an analogous manner to Lemma 5.2. We shall therefore omit the proof.

Lemma 4.3. For an appropriately small choice of $\tau>0$ depending on $p$ the following holds. If $v \in V$ $\tau$-regular then,

$$
\left\|f_{\mathbf{b}_{v}}\right\|_{p}^{p} \geqslant \gamma_{p}^{p}\left\|\mathbf{b}_{v}\right\|_{2}^{p}(1-\sqrt{\tau}) \text {. }
$$

Our next goal is to show that for small enough $\tau$ (to be set later in terms of $\eta$ and $p$ ), there is a significant fraction of vertices that are $\tau$-irregular. Let $S_{i r r}$ be the set of vertices that are $\tau$-irregular and let $\left|S_{i r r}\right|=\theta n$. By Lemma 4.1 we have,

$$
\begin{gathered}
\sum_{v \in S_{i r r}}\left\|\mathbf{b}_{v}\right\|_{2}^{2} \leqslant \gamma_{p}^{2} \theta^{1-2 / p} n \\
\Leftrightarrow \sum_{v \in V \backslash S_{i r r}}\left\|\mathbf{b}_{v}\right\|_{2}^{2} \geqslant n\left(1-\gamma_{p}^{2} \theta^{1-2 / p}\right)
\end{gathered}
$$

Also, from our initial assumption on the objective value of $\mathbf{b}$ given by Equation 12 we have,

$$
\begin{array}{rlr}
\gamma_{p}^{p}(1-\eta) n & \geqslant \quad \sum_{v \in V}\left\|f_{\mathbf{b}_{v}}\right\|_{p}^{p} \\
& \geqslant \quad \sum_{v \in V \backslash S_{i r r}}\left\|f_{\mathbf{b}_{v}}\right\|_{p}^{p} & \\
& \geqslant \quad \gamma_{p}^{p}(1-\sqrt{\tau}) \sum_{v \in V \backslash S_{i r r}}\left\|\mathbf{b}_{v}\right\|_{2}^{p} \quad \text { (by Lemma 4.3) } \\
& \geqslant \gamma_{p}^{p}(1-\sqrt{\tau})\left(\sum_{v \in V \backslash S_{i r r}}\left\|\mathbf{b}_{v}\right\|_{2}^{2}\right)^{\frac{p}{2}} \quad \text { (by Jensen's Inequality) } \\
& \geqslant \gamma_{p}^{p}(1-\sqrt{\tau}) n\left(1-\gamma_{p}^{2} \theta^{1-2 / p}\right) \quad \text { (by Equation 16) }
\end{array}
$$

From the above, choosing $0<\tau \leqslant \eta^{6}$, we obtain that $\theta>0$ is a constant depending only on $\eta$ and $p$. Therefore, at least $\theta>0$ fraction of the vertices are $\tau$-irregular where $0<\tau \leqslant \eta^{6}$ and $\theta=\theta(\eta, p)$. To complete the analysis of the soundness we shall show that the vector $\mathbf{b}$ can be decoded into a labeling for $\mathcal{L}$ that satisfies a significant fraction of the its edges, which shall contradict the soundness property of Theorem 3.5.

\section{Constructing a good labeling for $\mathcal{L}$}

The vector $\mathbf{b}$ shall be decoded into a labeling for the set of $\tau$-irregular vertices $S_{i r r}$. Observe that since $\left|S_{i r r}\right| \geqslant \theta n$, by the Weak Expansion property of Theorem 3.5,

$$
\left|E\left(S_{i r r}\right)\right| \geqslant\left(\theta^{2} / 2\right)|E|,
$$


where $E\left(S_{i r r}\right)$ is the set of edges induced by $S_{i r r}$. For every vertex $v \in S_{i r r}$, define,

$$
\Gamma_{0}(v):=\left\{i \in[M]|| \mathbf{b}(v, i) \mid \geqslant \frac{\tau}{2}\left\|\mathbf{b}_{v}\right\|_{2}\right\}
$$

and,

$$
\Gamma_{1}(v):=\left\{i \in[M]|| \mathbf{b}(v, i) \mid \geqslant \frac{\tau}{10 t}\left\|\mathbf{b}_{v}\right\|_{2}\right\},
$$

where $t=4^{u}$ is the parameter from Theorem 3.5. Clearly, for every vertex $v \in S_{i r r}$ :

$$
\emptyset \neq \Gamma_{0}(v) \subseteq \Gamma_{1}(v), \quad\left|\Gamma_{0}(v)\right| \leqslant \frac{4}{\tau^{2}}, \text { and }\left|\Gamma_{1}(v)\right| \leqslant \frac{100 t^{2}}{\tau^{2}} .
$$

Let $v$ be any vertex in $S_{i r r}$. Call an edge $e$ incident on $v$ to be "good" for $v$ if $\pi^{e, v}$ maps the set $\Gamma_{1}(v)$ injectively (one to one) into $[N]$. Using the smoothness property of Theorem 3.5 yields the following bound on the probability that a random edge incident on $v$ is "good":

$$
\operatorname{Pr}_{e \ni v}[e \text { is "good" for } v] \geqslant 1-\frac{\left|\Gamma_{1}(v)\right|^{2}}{J} \geqslant 1-\frac{10000 t^{4}}{\tau^{2} J}=: 1-\zeta \text {. }
$$

Since the graph of $\mathcal{L}$ is regular, this implies that the total number of edges induced by $S_{\text {irr }}$ that are not "good" for at least of the end points in $S_{i r r}$ is at most $2 \zeta|E|$. Let $E^{\prime} \subseteq E\left(S_{i r r}\right)$ be the set of edges induced by $S_{i r r}$ that are "good" for both endpoints. The above bounds combined with Equation 18 imply,

$$
\left|E^{\prime}\right| \geqslant\left(\frac{\theta^{2}}{2}-2 \zeta\right)|E|
$$

The following lemma shows that the folding constraints enforce a structural property on the sets $\Gamma_{0}(v)$ with respect to the edges in $E^{\prime}$.

Lemma 4.4. Let $e=(u, v)$ be any edge in $E^{\prime}$. Then $\pi^{e, u}\left(\Gamma_{0}(u)\right) \cap \pi^{e, v}\left(\Gamma_{0}(v)\right) \neq \emptyset$.

Proof. Clearly, $u$ and $v$ are $\tau$-irregular. Without loss of generality assume that $\left\|\mathbf{b}_{u}\right\|_{2} \geqslant\left\|\mathbf{b}_{v}\right\|$. Since $u$ is $\tau$-irregular, there is a coordinate $\left(u, i_{u}\right)\left(i_{u} \in[M]\right)$ such that $\left|\mathbf{b}\left(u, i_{u}\right)\right| \geqslant \tau\left\|\mathbf{b}_{u}\right\|_{2}$. By construction $i_{u} \in \Gamma_{0}(u)$.

Let $j_{0}:=\pi^{e, u}\left(i_{u}\right)$. Since $e \in E^{\prime},\left(\pi^{u, e}\right)^{-1}\left(j_{0}\right) \cap \Gamma_{1}(u)=\left\{i_{u}\right\}$. This implies that for all $i \in$ $\left(\pi^{u, e}\right)^{-1}\left(j_{0}\right)$ and $i \neq i_{u},|\mathbf{b}(u, i)|<\frac{\tau}{10 t}\left\|\mathbf{b}_{u}\right\|_{2}$. Moreover, from Theorem $3.5\left|\left(\pi^{u, e}\right)^{-1}\left(j_{0}\right)\right| \leqslant d$. Combining these observations yields,

$$
\left|\sum_{i \in\left(\pi^{u, e}\right)^{-1}\left(j_{0}\right)} \mathbf{b}(u, i)\right| \geqslant\left(\tau-t\left(\frac{\tau}{10 t}\right)\right)\left\|\mathbf{b}_{u}\right\|_{2}=\left(\frac{9 \tau}{10}\right)\left\|\mathbf{b}_{u}\right\|_{2} .
$$

We shall now show that $\left(\pi^{v, e}\right)^{-1}\left(j_{0}\right) \cap \Gamma_{0}(v) \neq \emptyset$, which would imply that $j_{0} \in \pi^{e, u}\left(\Gamma_{0}(u)\right) \cap$ $\pi^{e, v}\left(\Gamma_{0}(v)\right)$ thus completing the proof of the lemma.

For a contrapositive assume that $\left(\pi^{v, e}\right)^{-1}\left(j_{0}\right) \cap \Gamma_{0}(v)=\emptyset$. Moreover, since $e \in E^{\prime},\left(\pi^{v, e}\right)^{-1}\left(j_{0}\right) \cap$ $\Gamma_{1}(v) \leqslant 1$. This yields the following bound,

$$
\left|\sum_{i^{\prime} \in\left(\pi^{v, e}\right)^{-1}\left(j_{0}\right)} \mathbf{b}\left(v, i^{\prime}\right)\right| \leqslant\left(\frac{\tau}{2}+t\left(\frac{\tau}{10 t}\right)\right)\left\|\mathbf{b}_{v}\right\|_{2}=0.51 \tau\left\|\mathbf{b}_{v}\right\|_{2} .
$$


However, the folding constraints (Equation 8) imply that,

$$
\sum_{i \in\left(\pi^{u, e}\right)^{-1}\left(j_{0}\right)} \mathbf{b}(u, i)=\sum_{i^{\prime} \in\left(\pi^{v, e}\right)^{-1}\left(j_{0}\right)} \mathbf{b}\left(v, i^{\prime}\right),
$$

which is a contradiction to Equations 22 and 23 combined with $\left\|\mathbf{b}_{u}\right\|_{2} \geqslant\left\|\mathbf{b}_{v}\right\|_{2}>0$ (by the definition of $S_{i r r}$ ). This completes the proof of the lemma.

Let $L^{*}$ be a labeling to the vertices in $S_{i r r}$ constructed by independently and uniformly at random choosing a label from the set $\Gamma_{0}(v)$ for every vertex $v \in S_{i r r}$. By Lemma 4.4, every edge

$e=(u, v) \in E^{\prime}$ is satisfied with probability at least $\frac{1}{\left|\Gamma_{0}(u)\right|\left|\Gamma_{0}(v)\right|} \geqslant \frac{\tau^{4}}{16}$ (by Equation 19). Therefore, in expectation the total fraction $\Delta$ of edges satisfied is bounded by,

$$
\Delta \geqslant\left(\frac{\tau^{4}}{16}\right)\left(\frac{\theta^{2}}{2}-2 \zeta\right)
$$

Choosing $J>\left(4^{u}\right)^{5}$ and $u \gg 1$ large enough (depending on $\eta$ ) so that $\zeta \ll \theta$ one can ensure that $\Delta>2^{-c_{0} u}$ thereby yielding a contradiction to the soundness of Theorem 3.5. This completes the analysis of the NO case.

\section{$5 \quad$ Hardness Reduction for $\mathrm{QM}(p)$}

In this section we shall describe the NP-hardness reduction from Smooth Label Cover to the $L_{p}$ Quadratic Grothendieck Maximization Problem $\mathrm{QM}(p)$. The instance of $\mathrm{QM}(p)$ in our reduction is not explicitly given as a matrix. Instead we construct a set of coordinates and a quadratic form over any mapping from the set of coordinates to real numbers. A solution to this instance of $\mathrm{QM}(p)$ would be a mapping that maximizes the value of the quadratic form subject to the appropriate bound on the $p$ th norm of the mapping. While the initial construction would not ensure that the diagonal terms of the quadratic form are all 0 , our analysis shall prove that setting them to zero would not change the optimum of the instance significantly.

We start with an instance of Smooth Label Cover $\mathcal{L}\left(G(V, E), N, M,\left\{\pi^{v, e} \mid e \in E, v \in e\right\}\right)$ as given in Theorem 3.5. The parameters $J$ and $u$ shall be chosen appropriately for the soundness analysis of the reduction in Section 5.2. Let $n:=|V|$. Define the parameters $B$ and $D$ as follows:

$$
D:=d \cdot n^{12} \cdot|E|^{2} \cdot 2^{M} \quad \text { and } \quad B:=n^{10} \cdot|E|^{2} \cdot 2^{M}
$$

where $d$ is the degree of the (regular) graph $G$ of the instance $\mathcal{L}$ as given in Theorem 3.5. The first step of our construction is to define the coordinates.

Coordinates. For each vertex $v \in V$ there are $D$ sets of coordinates $C_{v}^{j}$ for $j=1, \ldots, D$. Each set $C_{v}^{j}$ consists of $2^{M}$ coordinates indexed by all elements of $\{-1,1\}^{M}$, and we denote the coordinate corresponding to $x \in\{-1,1\}^{M}$ by $C_{v}^{j}(x)$. Let,

$$
\mathcal{C}:=\bigcup_{v \in V} \bigcup_{j \in D} \bigcup_{x \in\{-1,1\}^{M}} C_{v}^{j}(x)
$$


denote the set of all coordinates.

Let $F: \mathcal{C} \mapsto \mathbb{R}$ be a mapping from the set of coordinates to real numbers. The quadratic form we construct shall be defined over $F$. Before we do so, we need to define some additional quantities. Given $F$ we define $f_{v}^{j}, f_{v}:\{-1,1\}^{M} \mapsto \mathbb{R}$ for all $v \in V$ and $j \in[D]$ by setting,

$$
\begin{aligned}
& f_{v}^{j}(x)=F\left(C_{v}^{j}(x)\right) \quad \text { and } \\
& f_{v}(x)=\mathbb{E}_{j \in[D]}\left[f_{v}^{j}(x)\right] \quad \forall x \in\{-1,1\}^{M} .
\end{aligned}
$$

In other words, $f_{v}$ is a point-wise average of $f_{v}^{j}$ over all $j \in[D]$. The $L_{q}$ norm of $F$ for $q \geqslant 1$ is given by:

$$
\|F\|_{q}:=\left(\mathbb{E}_{v \in V} \mathbb{E}_{j \in[D]}\left[\left\|f_{v}^{j}\right\|_{q}^{q}\right]\right)^{1 / q}
$$

where $\left\|f_{v}^{j}\right\|_{q}=\left(\mathbb{E}_{x \in\{-1,1\}^{M}}\left|f_{v}^{j}(x)\right|^{q}\right)^{1 / q}$. Now, since $\|f\|_{q}^{q}$ is a convex function for $q \geqslant 1$, we have by Jensen's inequality,

$$
\|F\|_{q} \geqslant\left(\mathbb{E}_{v \in V}\|f\|_{q}^{q}\right)^{1 / q} .
$$

We note that the functions $f_{v}(v \in V)$ can be written in their Fourier expansion with the basis functions $\chi_{S}(S \subseteq[M])$ with Fourier coefficients $\widehat{f}_{v}(S)$. Note that the Fourier coefficients are linear forms on the values of the function $f_{v}$. In our construction the quadratic form for $F$ shall be defined as a quadratic form over the Fourier coefficients. For convenience, we shall abuse notation to denote the Fourier coefficients corresponding to singleton sets $\{i\}(i \in[M])$ by $\widehat{f}_{v}(i)$.

The Quadratic Form. We define quadratic forms on $F: A_{\text {cons }}(F), A_{\text {dict }}(F)$ and $A_{\text {prel }}(F)$ in terms of the Fourier coefficients of the functions $f_{v}(v \in V)$ as follows.

$$
\begin{gathered}
A_{\text {cons }}(F):=-B \mathbb{E}_{e=(u, w)}\left[\sum_{i \in[M]}\left(\sum_{j \in \pi_{e, u}^{-1}(i)} \widehat{f}_{u}(j)-\sum_{j^{\prime} \in \pi_{e, w}^{-1}(i)} \widehat{f}_{w}\left(j^{\prime}\right)\right)^{2}\right] \\
-B \mathbb{E}_{v \in V}\left[\sum_{\substack{S \subseteq[M] \\
|S| \neq 1}} \widehat{f}_{v}(S)^{2}\right], \\
A_{\text {dict }}(F)=\mathbb{E}_{v \in V}\left[\sum_{j \in[M]} \widehat{f}_{u}(j)^{2}\right]
\end{gathered}
$$

and,

$$
A_{\text {prel }}(F)=A_{\text {cons }}(F)+A_{\text {dict }}(F) \text {. }
$$

In our reduction $F$ denotes a solution to our instance of $\mathrm{QM}(p)$ over which the quadratic form is defined. Therefore, $F$ satisfies the bound on its $p$-norm: $\|F\|_{p}=1$. 
We shall prove the completeness and soundness claims for the quadratic form $A_{\text {prel }}(F)$. However, we note that the $A_{\text {prel }}(F)$ may have non-zero diagonal terms. We address this issue in Section 5.3 wherein we show the existence of another quadratic form $A_{\text {fin }}(F)$ such that $\mid A_{\text {fin }}(F)-$ $A_{\text {prel }}(F) \mid \leqslant 100 / n$ for all $F$, which then suffices to prove Theorem 3.6.

Before we proceed, we state the instance of $\mathrm{QM}(p)$ as an optimization problem. The objective is to compute:

$$
\begin{array}{ll} 
& \max _{F: \mathcal{C} \mapsto \mathbb{R}} A_{\text {prel }}(F) \\
\text { s.t. } & \|F\|_{p}=1 .
\end{array}
$$

\subsection{Completeness}

Suppose the Smooth Label Cover instance $\mathcal{L}$ has a labeling $\sigma: V \mapsto[M]$ that satisfies all edges. Then construct the vector $F$ by defining $f_{v}^{j}$, for all $v \in V, j \in[D]$, as follows:

$$
f_{v}^{j}(x)=x(\sigma(v)), \quad \forall x \in\{-1,1\}^{M} .
$$

The above also implies that $f_{v}(x)=x(\sigma(v))$ for all $x \in\{-1,1\}^{M}$, i.e. $f_{v}$ is the 'dictator' function given by the $\sigma(v)$-th coordinate. Therefore, we obtain $\widehat{f}_{v}(\sigma(v))=1$ and $\widehat{f}_{v}(S)=0$ for all $S \neq$ $\{\sigma(v)\}$. Clearly, $\|F\|_{p}=1$ since $F$ is either 1 or -1 at any coordinate.

To analyze the value of $A_{\text {prel }}(F)$ we observe that $\pi_{e, u}(\sigma(u))=\pi_{e, w}(\sigma(w))$ for all edges $e=$ $(u, w)$, which along with the fact that $f_{u}(x)=x(\sigma(u))$ and $f_{w}(x)=x(\sigma(w))$ implies that $A_{\text {cons }}(F)=$ 0 . Also, $A_{\text {dict }}(F)=1$, which gives us that $A_{\text {prel }}(F)=1$.

\subsection{Soundness}

For a contradiction we assume that there is a vector $F$ such that $A_{\text {prel }}(F) \geqslant \gamma_{p}^{-2}(1+\eta)$ for some constant $\eta>0$. We shall show that this implies the existence of a labeling to $\mathcal{L}$ that satisfies a significant fraction of edges depending only on $\eta$.

The following is a straightforward upper bound on $A_{\text {dict }}(F)$ :

$$
\begin{aligned}
A_{\text {dict }}(F) & =\mathbb{E}_{v \in V}\left[\sum_{i \in[M]} \widehat{f}_{v}(i)^{2}\right] \\
& \leqslant \mathbb{E}_{v \in V}\left[\left\|f_{v}\right\|_{2}^{2}\right] \\
& \leqslant\left(\mathbb{E}_{v \in V}\left[\left\|f_{v}\right\|_{2}^{p}\right]\right)^{2 / p} \quad \text { (By Jensen's Inequality) } \\
& \left.\leqslant\left(\mathbb{E}_{v \in V}\left[\left\|f_{v}\right\|_{p}^{p}\right]\right)^{2 / p} \quad \text { (since }\|f\|_{p} \geqslant\|f\|_{2}\right) \\
& \leqslant\|F\|_{p}^{2} \leqslant 1 .
\end{aligned}
$$

For every $v \in V$ define: $a_{1}^{v}:=\sum_{|S| \neq 1, S \subseteq[M]}\left|\widehat{f}_{v}(S)\right|$ and $a_{2}^{v}:=\left(\sum_{|S| \neq 1, S \subseteq[M]}\left|\widehat{f}_{v}(S)\right|^{2}\right)^{1 / 2}$. Clearly, 
$a_{1}^{v} \leqslant 2^{M / 2} a_{2}^{v}$. Moreover, since $A_{\text {prel }}(F) \geqslant 0$, Equations 29,31 and 34 yield the following:

$$
\begin{aligned}
\frac{1}{B} & \geqslant \mathbb{E}_{v \in V}\left[\left(a_{2}^{v}\right)^{2}\right] \\
& \geqslant\left(\mathbb{E}_{v \in V}\left[a_{2}^{v}\right]\right)^{2} \\
& \geqslant\left(\mathbb{E}_{v \in V}\left[2^{-M / 2} a_{1}^{v}\right]\right)^{2} \\
\Rightarrow\left(\frac{2^{M}}{B}\right)^{1 / 2} & \geqslant \mathbb{E}_{v \in V}\left[a_{1}^{v}\right] \\
\Rightarrow\left(\frac{n^{2} 2^{M}}{B}\right)^{1 / 2} & \geqslant \max _{v \in V} a_{1}^{v}=: a_{\max }
\end{aligned}
$$

For the remainder of the analysis our focus shall be on the degree one Fourier spectrum of the functions $f_{v}$. Define, for every vertex $v: f_{v}^{=1}:=\sum_{i \in[M]} \widehat{f}_{v}(i) \chi_{\{i\}}$, i.e. the function obtained by taking only the degree one Fourier spectrum of $f_{v}$. Clearly, we have $\left|f_{v}(x)-f_{v}^{=1}(x)\right| \leqslant a_{\max }$ for all $x \in\{-1,1\}^{M}$ and $v \in V$. By the triangle inequality for $L_{q}, L_{q}^{\prime}$ norms (for $q, q^{\prime} \geqslant 1$ ) this implies,

$$
\begin{gathered}
\left|\left\|f_{v}\right\|_{q}-\left\|f_{v}^{=1}\right\|_{q}\right| \leqslant a_{\max } \quad \forall v \in V \\
\text { and, } \quad\left|\left(\mathbb{E}_{v \in V}\left[\left\|f_{v}\right\|_{q}^{q^{\prime}}\right]\right)^{1 / q^{\prime}}-\left(\mathbb{E}_{v \in V}\left[\left\|f_{v}^{=1}\right\|_{q}^{q^{\prime}}\right]\right)^{1 / q^{\prime}}\right| \leqslant a_{\max } .
\end{gathered}
$$

By our setting of $B, a_{\max }$ is at most $1 / n^{4}$. Since $p \geqslant 2$ is a fixed constant, setting $q=q^{\prime}=p$ in Equation 37 implies (for large enough $n$ ),

$$
\mathbb{E}_{v \in V}\left[\left\|f_{v}^{=1}\right\|_{p}^{p}\right] \leqslant \mathbb{E}_{v \in V}\left[\left\|f_{v}\right\|_{p}^{p}\right]+1 / n^{3} \leqslant\|F\|_{p}^{p}+1 / n^{3} \leqslant 1+1 / n^{3} . \quad \text { (By Equations 28, 33) }
$$

Similarly, using $q=p$ and $q^{\prime}=2$ in Equation 37 yields,

$$
\mathbb{E}_{v \in V}\left[\left\|f_{v}^{=1}\right\|_{p}^{2}\right] \leqslant 1+1 / n^{3}
$$

Using the above we obtain the following upper bound on the sum of the values $\left\|f_{v}^{=1}\right\|_{2}^{2}$ for small sets of vertices.

Lemma 5.1. Let $S \subseteq V$ be a set of size $\theta|V|=\theta$ n for some $0<\theta<1$. Then,

$$
\sum_{v \in S}\left\|f_{v}^{=1}\right\|_{2}^{2} \leqslant \theta^{1-2 / p_{n}}\left(1+1 / n^{3}\right)
$$

Proof. We need to upper bound $\beta$ where,

$$
\sum_{v \in S}\left\|f_{v}^{=1}\right\|_{2}^{2}=\beta n
$$

Note that the above implies that,

$$
\mathbb{E}_{v \in S}\left[\left\|f_{v}^{=1}\right\|_{2}^{2}\right]=\frac{\beta}{\theta} .
$$


Equation 38 yields $\mathbb{E}_{v \in V}\left[\left\|f_{v}^{=1}\right\|_{p}^{p}\right] \leqslant 1+1 / n^{3}$. This implies,

$$
\begin{aligned}
1+1 / n^{3} & \geqslant \quad \mathbb{E}_{v \in V}\left[\left\|f_{v}^{=1}\right\|_{2}^{p}\right] & & \left(\text { since }\|f\|_{p} \geqslant\|f\|_{2}\right) \\
& \geqslant \theta \mathbb{E}_{v \in S}\left[\left\|f_{v}^{=1}\right\|_{2}^{p}\right] & & \text { (by averaging) } \\
& \geqslant \theta\left(\mathbb{E}_{v \in S}\left[\left\|f_{v}^{=1}\right\|_{2}^{2}\right]\right)^{\frac{p}{2}} & & \text { (by Jensen's Inequality) } \\
& =\quad \theta\left(\frac{\beta}{\theta}\right)^{\frac{p}{2}} & & \text { (by Equation 41). }
\end{aligned}
$$

Therefore, $\beta \leqslant \theta^{1-2 / p}\left(1+1 / n^{3}\right)^{2 / p} \leqslant \theta^{1-2 / p}\left(1+1 / n^{3}\right)$, (since $p \geqslant 2$ ) which completes the proof of the lemma.

Before proceeding we choose a parameter $\tau>0$ which we shall later fix appropriately to depend only on $\eta$ and $p$. We now define the following set $V^{\prime} \subset V$ of vertices which have significantly large "mass" as follows.

$$
V^{\prime}:=\left\{v \in V \mid\left\|f_{v}^{=1}\right\|_{2}^{2} \geqslant 1 / n^{3}\right\}
$$

Further, define a subset $S_{i r r} \subseteq V^{\prime}$ as:

$$
S_{i r r}:=\left\{v \in V^{\prime} \quad \mid \exists i \in[M] \text { s.t. }\left|\widehat{f_{v}^{=1}}(i)\right|>\tau\left\|f_{v}^{=1}\right\|_{2}\right\}
$$

We shall refer to $S_{i r r}$ as the set of $\tau$-irregular vertices. Reusing notation for convenience, we assume that $\left|S_{i r r}\right|=\theta n$. Our goal is to show that $\theta$ is a significantly large constant depending on $\eta$ and $p$ (for an appropriate choice of $\tau$, again depending on $\eta$ and $p$ ). Lemma 5.1 applied to $S_{i r r}$ directly gives the following:

$$
\sum_{v \in S_{i r r}}\left\|f_{v}^{=1}\right\|_{2}^{2} \leqslant \theta^{1-2 / p} n\left(1+1 / n^{3}\right) .
$$

We also note that since $A_{\text {cons }}(F) \leqslant 0$ by definition, $A_{\text {prel }}(F) \leqslant A_{\text {dict }}(F)=\mathbb{E}_{v \in V}\left[\left\|f_{v}^{=1}\right\|_{2}^{2}\right]$. Therefore,

$$
\begin{aligned}
\gamma_{p}^{-2}(1+\eta) n & \leqslant \sum_{v \in V}\left\|f_{v}^{=1}\right\|_{2}^{2} \\
& =\sum_{v \in V^{\prime} \backslash S_{i r r}}\left\|f_{v}^{=1}\right\|_{2}^{2}+\sum_{v \in S_{i r r}}\left\|f_{v}^{=1}\right\|_{2}^{2}+\sum_{v \in V \backslash V^{\prime}}\left\|f_{v}^{=1}\right\|_{2}^{2} \\
& \leqslant \sum_{v \in V^{\prime} \backslash S_{i r r}}\left\|f_{v}^{=1}\right\|_{2}^{2}+\theta^{1-2 / p} n\left(1+1 / n^{3}\right)+1 / n^{2},
\end{aligned}
$$

where we used Equation 44 along with the bound $\sum_{v \in V \backslash V^{\prime}}\left\|f_{v}^{=1}\right\|_{2}^{2} \leqslant n \cdot\left(1 / n^{3}\right)=1 / n^{2}$. Rearranging the above we obtain,

$$
\sum_{v \in V^{\prime} \backslash S_{i r r}}\left\|f_{v}^{=1}\right\|_{2}^{2} \geqslant \gamma_{p}^{-2}(1+\eta) n-\theta^{1-2 / p_{n}} n\left(1+1 / n^{3}\right)-1 / n^{2} .
$$

To show that $\theta$ is large, we need to upper bound the LHS of the above equation. For this we use the following lemma which follows from Lemma 3.3.

Lemma 5.2. For an appropriately small choice of $\tau>0$ depending on $p$ the following holds. For all vertices $v \in V^{\prime} \backslash S_{i r r}$

$$
\left\|f_{v}^{=1}\right\|_{p}^{2} \geqslant \gamma_{p}^{2}\left\|f_{v}^{=1}\right\|_{2}^{2}(1-\sqrt{\tau}) \text {. }
$$


Proof. We consider probability space given by the uniform distribution over $x \in\{-1,1\}^{M}$. Defining $X_{i}:=x_{i}\left(\frac{\widehat{f}_{v}(i)}{\left\|f_{v}^{=1}\right\|_{2}}\right)$ we observe $X_{i}$ are Bernoulli variables with $\mathbb{E}\left[X_{i}\right]=0$ for all $1 \leqslant i \leqslant n$ and $\sum_{j=1}^{n} \mathbb{E}\left[X_{j}^{2}\right]=1$. Moreover, since $v \in V^{\prime} \backslash S_{i r r}, \sum_{j=1}^{n} \mathbb{E}\left[\left|X_{j}\right|^{3}\right] \leqslant \tau$. Applying Lemma 3.3 and observing that for small enough $\tau>0$ depending on $p,\left(1-4 \tau(\log (1 / \tau))^{p / 2}\right)^{2} \geqslant(1-\sqrt{\tau})$, we obtain the desired bound.

The above analysis yields the following sequence of inequalities which gives us a lower bound on $\theta$ depending on $\eta$.

$$
\begin{aligned}
\left(1+1 / n^{3}\right) n & \geqslant \sum_{v \in V}\left\|f_{v}^{=1}\right\|_{p}^{2} \quad(\text { By Equation 39) } \\
& \geqslant \sum_{v \in V^{\prime} \backslash S_{i r r}}\left\|f_{v}^{=1}\right\|_{p}^{2} \\
& \geqslant \gamma_{p}^{2}(1-\sqrt{\tau}) \sum_{v \in V^{\prime} \backslash S_{i r r}}\left\|f_{v}^{=1}\right\|_{2}^{2} \quad(\text { By Lemma 5.2) } \\
& \geqslant\left(\gamma_{p}^{2}(1-\sqrt{\tau})\right)\left(\gamma_{p}^{-2}(1+\eta) n-\theta^{1-2 / p} n\left(1+1 / n^{3}\right)-1 / n^{2}\right) \\
& =n(1-\sqrt{\tau})\left(1+\eta-\gamma_{p}^{2} \theta^{1-2 / p}\left(1+1 / n^{3}\right)-\gamma_{p}^{2} / n\right)
\end{aligned}
$$

Choosing $0<\tau \leqslant \eta^{6}$ in the above inequality, and using the fact that $p>2$ is a fixed constant, for large enough $n$, we obtain that $\theta>0$ is (at least) a positive constant depending on $\eta$ and $p$. Therefore, $S_{i r r}$ contains at least a constant fraction of vertices in $V$. To complete the soundness analysis we shall use this to obtain a substantially good labeling to the instance $\mathcal{L}$.

\subsubsection{Recovering a good labeling to $\mathcal{L}$}

$F$ shall be decoded into a labeling for the set of $\tau$-irregular vertices $S_{i r r}$. Observe that since $\left|S_{i r r}\right| \geqslant$ $\theta n$, by the Weak Expansion property of Theorem 3.5,

$$
\left|E\left(S_{i r r}\right)\right| \geqslant\left(\theta^{2} / 2\right)|E|,
$$

where $E\left(S_{i r r}\right)$ is the set of edges induced by $S_{i r r}$. For every vertex $v \in S_{i r r}$, define,

$$
\Gamma_{0}(v):=\left\{i \in[M]|| \widehat{f_{v}^{=1}}(i) \mid \geqslant \frac{\tau}{2}\left\|f_{v}^{=1}\right\|_{2}\right\}
$$

and,

$$
\Gamma_{1}(v):=\left\{i \in[M]|| \widehat{f_{v}^{=1}}(i) \mid \geqslant \frac{\tau}{10 t}\left\|f_{v}^{=1}\right\|_{2}\right\}
$$

where $t=4^{u}$ is the parameter from Theorem 3.5. Clearly, for every vertex $v \in S_{i r r}$ :

$$
\emptyset \neq \Gamma_{0}(v) \subseteq \Gamma_{1}(v), \quad\left|\Gamma_{0}(v)\right| \leqslant \frac{4}{\tau^{2}}, \text { and }\left|\Gamma_{1}(v)\right| \leqslant \frac{100 t^{2}}{\tau^{2}}
$$

Let $v$ be any vertex in $S_{i r r}$. Call an edge $e$ incident on $v$ to be "good" for $v$ if $\pi^{e, v}$ maps the set $\Gamma_{1}(v)$ injectively (one to one) into $[N]$. Using the smoothness property of Theorem 3.5 yields the 
following bound on the probability that a random edge incident on $v$ is "good":

$$
\mathbf{P r}_{e \ni v}[e \text { is "good" for } v] \geqslant 1-\frac{\left|\Gamma_{1}(v)\right|^{2}}{J} \geqslant 1-\frac{10000 t^{4}}{\tau^{4} J}=: 1-\zeta \text {. }
$$

Since the graph of $\mathcal{L}$ is regular, this implies that the total number of edges induced by $S_{\text {irr }}$ that are not "good" for at least one of the end points in $S_{i r r}$ is at most $2 \zeta|E|$. Let $E^{\prime} \subseteq E\left(S_{i r r}\right)$ be the set of edges induced by $S_{i r r}$ that are "good" for both endpoints. The above bounds combined with Equation 47 imply,

$$
\left|E^{\prime}\right| \geqslant\left(\frac{\theta^{2}}{2}-2 \zeta\right)|E|
$$

The following lemma shows that the constraints given by Equation 29 enforce a structural property on the sets $\Gamma_{0}(v)$ with respect to the edges in $E^{\prime}$.

Lemma 5.3. Let $e=(u, v)$ be any edge in $E^{\prime}$. Then $\pi^{e, u}\left(\Gamma_{0}(u)\right) \cap \pi^{e, v}\left(\Gamma_{0}(v)\right) \neq \emptyset$.

Proof. Clearly, $u$ and $v$ are in $S_{i r r}$. Without loss of generality assume that $\left\|f_{u}^{=1}\right\|_{2} \geqslant\left\|f_{v}^{=1}\right\| \geqslant 1 / n^{3}$ where the lower bound is because $S_{i r r}$ is a subset of $V^{\prime}$. Since $u$ is $\tau$-irregular, there is exists $i_{u} \in[M]$ such that $\left|\widehat{f_{u}^{=1}}\left(i_{u}\right)\right| \geqslant \tau\left\|f_{u}^{=1}\right\|_{2}$. By construction $i_{u} \in \Gamma_{0}(u)$.

Let $j_{0}:=\pi^{e, u}\left(i_{u}\right)$. Since $e \in E^{\prime},\left(\pi^{u, e}\right)^{-1}\left(j_{0}\right) \cap \Gamma_{1}(u)=\left\{i_{u}\right\}$. This implies that for all $i \in$ $\left(\pi^{u, e}\right)^{-1}\left(j_{0}\right)$ and $i \neq i_{u},\left|\widehat{f_{u}^{=1}}(i)\right|<\frac{\tau}{10 t}\left\|f_{u}^{=1}\right\|_{2}$. Moreover, from Theorem $3.5\left|\left(\pi^{u, e}\right)^{-1}\left(j_{0}\right)\right| \leqslant t$. Combining these observations yields,

$$
\left|\sum_{i \in\left(\pi^{u, e}\right)^{-1}\left(j_{0}\right)} \widehat{f_{u}^{=1}}(i)\right| \geqslant\left(\tau-t\left(\frac{\tau}{10 t}\right)\right)\left\|f_{u}^{=1}\right\|_{2}=\left(\frac{9 \tau}{10}\right)\left\|f_{u}^{=1}\right\|_{2} .
$$

We shall now show that $\left(\pi^{v, e}\right)^{-1}\left(j_{0}\right) \cap \Gamma_{0}(v) \neq \emptyset$, which would imply that $j_{0} \in \pi^{e, u}\left(\Gamma_{0}(u)\right) \cap$ $\pi^{e, v}\left(\Gamma_{0}(v)\right)$ thus completing the proof of the lemma.

For a contrapositive assume that $\left(\pi^{v, e}\right)^{-1}\left(j_{0}\right) \cap \Gamma_{0}(v)=\emptyset$. Moreover, since $e \in E^{\prime},\left(\pi^{v, e}\right)^{-1}\left(j_{0}\right) \cap$ $\Gamma_{1}(v) \leqslant 1$. This yields the following bound,

$$
\left|\sum_{i^{\prime} \in\left(\pi^{v, e}\right)^{-1}\left(j_{0}\right)} \widehat{f_{v}^{=1}}\left(i^{\prime}\right)\right| \leqslant\left(\frac{\tau}{2}+t\left(\frac{\tau}{10 t}\right)\right)\left\|f_{v}^{=1}\right\|_{2}=0.51 \tau\left\|f_{v}^{=1}\right\|_{2} \leqslant 0.51 \tau\left\|f_{u}^{=1}\right\|_{2} .
$$

Noting that for any vertex $w \in V$ and $i \in[M], \widehat{f_{w}^{=1}}(i)=\widehat{f_{w}}(i)$, the constraints given by Equation 29 imply,

$$
\left|\sum_{i \in\left(\pi^{u, e}\right)^{-1}\left(j_{0}\right)} \widehat{f_{u}^{=1}}(i)-\sum_{i^{\prime} \in\left(\pi^{v, e}\right)^{-1}\left(j_{0}\right)} \widehat{f_{\bar{v}}^{=1}}\left(i^{\prime}\right)\right| \leqslant \frac{|E|}{\sqrt{B}} \leqslant \frac{1}{n^{5}}
$$

by our setting of $B$. Since $\tau>0$ is a constant, this is a contradiction to Equations 51 and 52 combined with $\left\|f_{u}\right\|_{2} \geqslant 1 / n^{3}$, for large enough $n$. This completes the proof of the lemma. 
Let $L^{*}$ be a labeling to the vertices in $S_{i r r}$ constructed by independently and uniformly at random choosing a label from the set $\Gamma_{0}(v)$ for every vertex $v \in S_{i r r}$. By Lemma 5.3, every edge $e=(u, v) \in E^{\prime}$ is satisfied with probability at least $\frac{1}{\left|\Gamma_{0}(u)\right|\left|\Gamma_{0}(v)\right|} \geqslant \frac{\tau^{4}}{16}$ (by Equation 48). Therefore, in expectation the total fraction $\Delta$ of edges satisfied is lower bounded by,

$$
\Delta \geqslant\left(\frac{\tau^{4}}{16}\right)\left(\frac{\theta^{2}}{2}-2 \zeta\right) .
$$

Choosing $J>\left(4^{u}\right)^{5}$ and $u \gg 1$ large enough (depending on $\eta$ ) so that $\zeta \ll \theta$ one can ensure that $\Delta>2^{-c_{0} u}$ thereby yielding a contradiction to the soundness of Theorem 3.5. This completes the analysis of the soundness case.

\subsection{Removing the diagonal terms}

In this section we show that there is a quadratic form $A_{\text {fin }}(F)$ with no diagonal entries, such $\left|A_{\text {fin }}(F)-A_{\text {prel }}(F)\right| \leqslant 1 / n$ for $F$ such that $\|F\|_{p}=1$. Our reduction would output a slightly scaled version of $A_{\text {fin }}(F)$ as the quadratic form to ensure that in the completeness case of Theorem 3.6 the optimum is at least 1 . Since the scaling is by at most a factor of $(1+1 / n)$ the error induced in the soundness case can be absorbed in the constant $\epsilon>0$ of Theorem 3.6, thus completing its proof. The rest of this section is devoted to computing $A_{\text {fin }}(F)$.

We first note that the source of the diagonal terms in $A_{\text {prel }}(F)$ are terms of the form $\widehat{f}_{v}(S)^{2}$. Moreover, for any vertex $v \in V$ and set $S \subseteq[M]$ we have,

$$
\begin{aligned}
\widehat{f_{v}}(S)^{2} & =\left(\mathbb{E}_{j \in[D]}\left[\widehat{f_{v}^{j}}(S)\right]\right)^{2} \\
& =\frac{1}{D^{2}} \cdot \sum_{j_{1}, j_{2} \in[D]} \widehat{f_{v}^{j_{1}}}(S) \widehat{f_{v}^{j_{2}}}(S) \\
& =\frac{1}{D^{2}} \cdot \sum_{\substack{j_{1}, j_{2} \in[D] \\
j_{1} \neq j_{2}}} \widehat{f_{v}^{j_{1}}}(S) \widehat{f_{v}^{j_{2}}}(S)+\frac{1}{D^{2}} \cdot \sum_{j \in[D]} \widehat{f_{v}^{j}}(S)^{2} .
\end{aligned}
$$

Note that the diagonal terms are a consequence of only the second term in the expression in Equation 53. Therefore, the quadratic form $\widehat{f}_{v}(S)^{2}-T_{v}(S)$ has no diagonal terms where,

$$
T_{v}(S):=\frac{1}{D^{2}} \cdot \sum_{j \in[D]} \widehat{f_{v}^{j}}(S)^{2},
$$


for $v \in V$ and $S \subseteq[M]$. Note that $T_{v}(S) \geqslant 0$. In addition we have,

$$
\begin{aligned}
\mathbb{E}_{v \in V}\left[\sum_{S \subseteq[M]} T_{v}(S)\right] & =\mathbb{E}_{v \in V}\left[\sum_{S \subseteq[M]} \frac{1}{D^{2}} \cdot \sum_{j \in[D]} \widehat{f_{v}^{j}}(S)^{2}\right] \\
& =\mathbb{E}_{v \in V}\left[\frac{1}{D^{2}} \sum_{j \in[D]} \sum_{S \subseteq[M]} \widehat{f_{v}^{j}}(S)^{2}\right] \\
& =\frac{1}{D}\left(\mathbb{E}_{v \in V} \mathbb{E}_{j \in[D]}\left[\sum_{S \subseteq[M]} \widehat{f_{v}^{j}}(S)^{2}\right]\right) \\
& =\frac{1}{D}\left(\mathbb{E}_{v \in V} \mathbb{E}_{j \in[D]}\left[\left\|f_{v}^{j}\right\|_{2}^{2}\right]\right) \\
& =\frac{1}{D}\|F\|_{2}^{2} \leqslant \frac{1}{D}\|F\|_{p}^{2}=\frac{1}{D} .
\end{aligned}
$$

Observing that the graph of $\mathcal{L}$ is regular, it can be seen that $A_{\text {cons }}(F)$ can be written as,

$$
A_{\text {cons }}(F)=-B \mathbb{E}_{v \in V}\left[2 \cdot \sum_{i \in[M]} \widehat{f}_{v}^{2}(i)+\sum_{\substack{S \subseteq[M] \\|S| \neq 1}} \widehat{f}_{v}(S)^{2}\right]+A_{\text {cross }}(F)
$$

where $A_{\text {cross }}(F)$ is a quadratic form involving terms of the type $\widehat{f}_{u}(i) \widehat{f_{w}}(j)$ where $u, w \in V, u \neq w$ and $i, j \in[M]$, and therefore does not contribute any diagonal terms. Defining $A_{\text {cons }}^{*}(F)$ as,

$$
A_{\text {cons }}^{*}(F)=A_{\text {cons }}(F)+B \mathbb{E}_{v \in V}\left[2 \cdot \sum_{\substack{S^{\prime} \subseteq[M] \\\left|S^{\prime}\right|=1}} T_{v}\left(S^{\prime}\right)+\sum_{\substack{S \subseteq[M] \\|S| \neq 1}} T_{v}(S)\right]
$$

we see that $A_{\text {cons }}^{*}(F)$ does not contain any diagonal terms. Similarly, defining $A_{\text {dict }}^{*}(F)$ as,

$$
A_{\text {dict }}^{*}(F)=A_{\text {dict }}(F)-\mathbb{E}_{v \in V}\left[\sum_{\substack{S^{\prime} \subseteq[M] \\\left|S^{\prime}\right|=1}} T_{v}\left(S^{\prime}\right)\right]
$$

it can be seen that $A_{\text {dict }}^{*}(F)$ does not contain any diagonal terms. Let $A_{\text {fin }}(F)=A_{\text {cons }}^{*}(F)+A_{\text {dict }}^{*}(F)$. Using Equations 55, 56 and 57 it is easy to see that,

$$
\left|A_{\text {cons }}^{*}(F)-A_{\text {cons }}(F)\right| \leqslant \frac{2 B}{D} \quad \text { and } \quad\left|A_{\text {dict }}^{*}(F)-A_{\text {dict }}(F)\right| \leqslant \frac{1}{D} .
$$

Therefore we obtain the desired bound,

$$
\left|A_{\text {fin }}(F)-A_{\text {prel }}(F)\right| \leqslant \frac{2 B+1}{D} \leqslant 1 / n,
$$

by our setting of $B$ and $D$. 


\section{Approximation for $\mathrm{QM}(p)$}

Let $A=\left(a_{i j}\right)_{i, j=1}^{n}$ be an $n \times n$ symmetric matrix with diagonal entries all zero, given as an instance of $\mathrm{QM}(p)$ for a fixed $p \geqslant 2$. We have,

$$
\operatorname{Val}_{p}(A)=\max \left\{\sum_{i, j=1}^{n} a_{i j} x_{i} x_{j}:\left\{x_{1}, \ldots, x_{n}\right\} \subseteq \mathbb{R}, \sum_{i=1}^{n}\left|x_{i}\right|^{p} \leqslant 1\right\} .
$$

As shown in Kindler et al. the above can be relaxed to the following convex program,

$$
\operatorname{Vec}_{p}(A)=\max \left\{\sum_{i, j=1}^{n} a_{i j}\left\langle u_{i}, u_{j}\right\rangle:\left\{u_{1}, \ldots, u_{n}\right\} \subseteq L_{2}, \sum_{i=1}^{n}\left\|u_{i}\right\|_{2}^{p} \leqslant 1\right\} .
$$

Let $v_{1}, \ldots, v_{n}$ denote an optimal solution to the above convex program. Let $h_{1}, \ldots, h_{n}$ be mean zero Gaussian random variables obtained by defining $h_{i}:=\left\langle G, v_{i}\right\rangle(1 \leqslant i \leqslant n)$, where $G$ is a random Gaussian vector in the space spanned by $v_{1}, \ldots, v_{n}$. It is easy to see that the following properties are satisfied.

$$
\sum_{i=1}^{n}\left(\mathbb{E}\left[h_{i}^{2}\right]\right)^{p / 2} \leqslant 1 \quad \text { and } \mathbb{E}\left[\sum_{i, j=1}^{n} a_{i j} h_{i} h_{j}\right]=\operatorname{Vec}_{p}(A) .
$$

Now we simply note that,

$$
\begin{aligned}
& \operatorname{Vec}_{p}(A)=\mathbb{E}\left[\sum_{i, j=1}^{n} a_{i j} h_{i} h_{j}\right] \\
& =\mathbb{E}\left[\left(\sum_{k=1}^{n}\left|h_{k}\right|^{p}\right)^{2 / p}\left[\sum_{i, j=1} a_{i j}\left(\frac{h_{i}}{\left(\sum_{k=1}^{n}\left|h_{k}\right|^{p}\right)^{1 / p}}\right)\left(\frac{h_{j}}{\left(\sum_{k=1}^{n}\left|h_{k}\right|^{p}\right)^{1 / p}}\right)\right]\right] \\
& \leqslant \mathbb{E}\left[\left(\sum_{k=1}^{n}\left|h_{k}\right|^{p}\right)^{2 / p} \cdot \operatorname{Val}_{p}(A)\right] \quad\left(\text { By Definition of } \operatorname{Val}_{p}(A)\right) \\
& \leqslant\left(\sum_{k=1}^{n} \mathbb{E}\left[\left|h_{k}\right|^{p}\right]\right)^{2 / p} \operatorname{Val}_{p}(A) \quad(\text { By Jensens Inequality and since } p \geqslant 2) \\
& =\left(\sum_{k=1}^{n} \gamma_{p}^{p}\left(\mathbb{E}\left[h_{k}^{2}\right]\right)^{p / 2}\right)^{2 / p} \operatorname{Val}_{p}(A) \quad \text { (By Definition of } \gamma_{p} \text { and since } h_{k} \text { is Gaussian) } \\
& \leqslant \gamma_{p}^{2} \operatorname{Val}_{p}(A) \quad(\text { By Equation 61) }
\end{aligned}
$$

which is the upper bound we wanted. Note that the upper bound is obtained directly without rounding the vectors. To complete the proof of Theorem 3.7 we need to demonstrate a polynomial time rounding algorithm that extracts a $\gamma_{p}^{2}(1+\delta)$ approximate solution $x_{1}^{*}, \ldots, x_{n}^{*}$ to $\operatorname{Val}_{p}(A)$ from the vectors $v_{1}, \ldots, v_{n}$ for any constant $\delta>0$. This shall be our goal in the remainder of the section.

Before we do so we can first assume without the loss of generality that

$$
\left|a_{12}\right|=1=\max _{1 \leqslant i, j \leqslant n}\left|a_{i j}\right|,
$$


by appropriately relabeling the entries of the matrix $A$ and scaling them. Setting $x_{1}=1 / 2$ and $x_{2}=a_{12} /\left|a_{12}\right|$ and $x_{3}, \ldots, x_{n}=0$ we obtain that

$$
\operatorname{Vec}_{p}(A) \geqslant \operatorname{Val}_{p}(A) \geqslant 1 / 4
$$

The following is the rounding algorithm that we shall analyze.

$\operatorname{Algorithm~Round}\left(A,\left\{v_{1}, \ldots, v_{n}\right\}\right)$ :

1. Let $T:=n^{22}$. Sample $T$ random Gaussian vectors $G_{1}, \ldots, G_{T}$ in the span of $v_{1}, \ldots, v_{n}$.

2. Define random variables $z_{i}^{(t)}:=\left\langle G_{t}, v_{i}\right\rangle$ for all $1 \leqslant t \leqslant T$ and $1 \leqslant i \leqslant n$. In addition define

$$
x_{i}^{(t)}:=\frac{z_{i}^{(t)}}{\left(\sum_{k=1}^{n}\left|z_{j}^{(t)}\right|^{p}\right)^{1 / p}}
$$

and,

$$
\Delta_{t}:=\sum_{i, j=1}^{n} a_{i j} x_{i}^{(t)} x_{j}^{(t)} .
$$

3. Let $t^{*} \in\{1, \ldots, T\}$ be such that $\Delta_{t^{*}}=\max _{1 \leqslant t \leqslant T} \Delta_{t}$. Output $x_{1}^{*}, \ldots, x_{n}^{*}$ as the solution where $x_{i}^{*}=x_{i}^{\left(t^{*}\right)}$ for $1 \leqslant i \leqslant n$.

Let $\mathbb{E}_{t}$ denote the expectation over the uniformly at random choice of $t$ from $1, \ldots, T$. We begin with the following lemma.

Lemma 6.1. Given the random variables constructed in the procedure $\operatorname{Round}\left(A,\left\{v_{1}, \ldots, v_{n}\right\}\right)$, with probability at least $1-1 / n^{8}$ over the choice of $G_{1}, \ldots, G_{T}$ the following inequality holds,

$$
\sum_{i, j=1}^{n} a_{i j} \mathbb{E}_{t}\left[z_{i}^{(t)} z_{j}^{(t)}\right] \geqslant\left(1-8 / n^{2}\right) \operatorname{Vec}_{p}(A)
$$

Proof. We begin by ignoring the terms corresponding to pairs $i, j(1 \leqslant i, j \leqslant n)$ such that $\left\langle v_{i}, v_{j}\right\rangle$ is very small. Formally, Let $R:=\left\{(i, j) \in[n] \times[n]\left|\left\langle v_{i}, v_{j}\right\rangle\right| \geqslant 1 / n^{4}\right\}$. We have,

$$
\begin{aligned}
\left|\sum_{(i, j) \in R} a_{i j}\left\langle v_{i}, v_{j}\right\rangle-\sum_{i, j=1}^{n} a_{i j}\left\langle v_{i}, v_{j}\right\rangle\right| & \leqslant \sum_{(i, j) \notin R}\left|a_{i j}\left\langle v_{i}, v_{j}\right\rangle\right| \\
& \leqslant \sum_{(i, j) \notin R}\left|a_{i j}\right|\left(\frac{1}{n^{4}}\right) \\
& \leqslant n^{2}\left(\frac{1}{n^{4}}\right)=1 / n^{2} . \quad \text { (By Equation 62) }
\end{aligned}
$$

Now consider any $(i, j) \in R$. As before, we have Gaussian random variables $h_{i}$ and $h_{j}$ such that $\mathbb{E}\left[h_{i} h_{j}\right]=\left\langle v_{i}, v_{j}\right\rangle$. Moreover, since $(i, j) \in R,\left|\mathbb{E}\left[h_{i} h_{j}\right]\right| \geqslant 1 / n^{4}$. We also need a bound on the 
variance of $h_{i} h_{j}$. Clearly, $\operatorname{Var}\left[h_{i} h_{j}\right] \leqslant \mathbb{E}\left[h_{i}^{2} h_{j}^{2}\right]$. Also, from Equation 61 we have that $\mathbb{E}\left[h_{i}^{2}\right], \mathbb{E}\left[h_{j}^{2}\right] \leqslant$ 1. Therefore, $\mathbb{E}\left[h_{i}^{2} h_{j}^{2}\right]$ is upper bounded by $\mathbb{E}\left[g^{4}\right]=3$ where $g$ is a standard Gaussian variable with variance 1 . We note that over the choice of $G_{1}, \ldots, G_{T}$, the random variables $z_{i}^{(t)} z_{j}^{(t)}$ are identically distributed as $h_{i} h_{j}$ for all $1 \leqslant t \leqslant T$. Moreover, since $G_{1}, \ldots, G_{T}$ are independent Gaussian vectors, the random variables $z_{i}^{(t)} z_{j}^{(t)}$ are also independent for $1 \leqslant t \leqslant T$. Therefore,

$$
\operatorname{Var}\left[\mathbb{E}_{t}\left[z_{i}^{(t)} z_{j}^{(t)}\right]\right] \leqslant \frac{\operatorname{Var}\left[h_{i} h_{j}\right]}{T} \leqslant \frac{1}{n^{20}}
$$

by our choice of $T$ and where the variance is over the choice of $G_{1}, \ldots, G_{T}$. Moreover, since $\mathbb{E}\left[\mathbb{E}_{t}\left[z_{i}^{(t)} z_{j}^{(t)}\right]\right]=\mathbb{E}\left[h_{i} h_{j}\right]$, we have the following bound using Chebyshev's inequality.

$$
\operatorname{Pr}\left[\left|\mathbb{E}_{t}\left[z_{i}^{(t)} z_{j}^{(t)}\right]-\mathbb{E}\left[h_{i} h_{j}\right]\right| \geqslant 1 / n^{5}\right] \leqslant 1 / n^{10}
$$

Since the above analysis holds for all pairs $(i, j) \in R$, using a union bound over all pairs the above implies that with probability at least $1-1 / n^{8}$, the following holds,

$$
\left|\sum_{(i, j) \in R} a_{i j} \mathbb{E}_{t}\left[z_{i}^{(t)} z_{j}^{(t)}\right]-\sum_{(i, j) \in R} a_{i j} \mathbb{E}\left[h_{i} h_{j}\right]\right| \leqslant\left(1 / n^{5}\right) \sum_{(i, j) \in R} a_{i j} \leqslant 1 / n^{3},
$$

where the final inequality is obtained using Equation 62. Combining the above with Equation 65 implies that the following holds with probability at least $1-1 / n^{8}$,

$$
\left|\sum_{i, j=1}^{n} a_{i j} \mathbb{E}_{t}\left[z_{i}^{(t)} z_{j}^{(t)}\right]-\sum_{i, j=1}^{n} a_{i j}\left\langle v_{i}, v_{j}\right\rangle\right| \leqslant 1 / n^{2}+1 / n^{3} \leqslant 2 / n^{2}
$$

This implies that with probability at least $1-1 / n^{8}$,

$$
\sum_{i, j=1}^{n} a_{i j} \mathbb{E}_{t}\left[z_{i}^{(t)} z_{j}^{(t)}\right] \geqslant \operatorname{Vec}_{p}(A)-2 / n^{2} \geqslant\left(1-8 / n^{2}\right) \operatorname{Vec}_{p}(A),
$$

where the last inequality follows from Equation 63. This completes the proof of the lemma.

The next lemma also proves a similar bound for the $p$ th moments of the variables Gaussian variables $h_{i}$.

Lemma 6.2. With probability at least $1-1 / n^{8}$ over the choice of $G_{1}, \ldots, G_{T}$ the following holds for every $i=1, \ldots, n$.

$$
\left|\mathbb{E}_{t}\left[\left|z_{i}^{(t)}\right|^{p}\right]-\mathbb{E}\left[\left|h_{i}\right|^{p}\right]\right| \leqslant 1 / n^{4}
$$

Proof. Let us fix $i \in\{1, \ldots, n\}$ for the moment. As noted before, over the choice of $G_{1}, \ldots, G_{T}$ the random variables $z_{i}^{(t)}, 1 \leqslant t \leqslant T$, are independent random variables distributed identically to $h_{i}$. Now we have,

$$
\operatorname{Var}\left[\left|h_{i}\right|^{p}\right] \leqslant \mathbb{E}\left[\left|h_{i}\right|^{p}\right] \leqslant \gamma_{p}^{p}\left(\mathbb{E}\left[\left|h_{i}^{2}\right|\right]\right)^{2 / p} \leqslant \gamma_{p}^{p},
$$


where the second last inequality is by the definition of $\gamma_{p}$ and the last inequality uses Equation 61. Since $z_{i}^{(t)}$ are independent for $1 \leqslant t \leqslant T$, this implies,

$$
\operatorname{Var}\left[\mathbb{E}_{t}\left[\left|z_{i}^{(t)}\right|^{p}\right]\right] \leqslant \frac{\operatorname{Var}\left[\left|h_{i}\right|^{p}\right]}{T} \leqslant \frac{\gamma_{p}^{p}}{n^{22}} \leqslant \frac{1}{n^{20}}
$$

for large enough $n$. Therefore, by Chebyshev's inequality we obtain,

$$
\operatorname{Pr}\left[\left|\mathbb{E}_{t}\left[\left|z_{i}^{(t)}\right|^{p}\right]-\mathbb{E}\left[\left|h_{i}\right|^{p}\right]\right| \geqslant 1 / n^{5}\right] \leqslant 1 / n^{10}
$$

Taking a union bound over all $i=1, \ldots, n$ and rearranging Equation 71 proves the lemma.

We are now ready to prove the desired bounds on the performance of the rounding algorithm $\operatorname{Round}\left(A,\left\{v_{1}, \ldots, v_{n}\right\}\right)$. For this we need to prove a upper bound on $\operatorname{Vec}_{p}(A)$ in terms of $\Delta^{*}$. This is shown through the following series of inequalities implied by the two previous lemmas whose conditions hold with probability at least $1-2 / n^{8}$.

$$
\begin{aligned}
\left(1-8 / n^{2}\right) \operatorname{Vec}_{p}(A) & \leqslant \mathbb{E}_{t}\left[\sum_{i, j=1}^{n} a_{i j} z_{i}^{(t)} z_{j}^{(t)}\right] \quad(\text { By Lemma 6.1) } \\
& \leqslant \mathbb{E}_{t}\left[\left(\sum_{k=1}^{n}\left|z_{k}^{(t)}\right|^{p}\right)^{2 / p} \sum_{i, j=1}^{n} a_{i j}\left(\frac{z_{i}^{(t)}}{\left(\sum_{k=1}^{n}\left|z_{k}^{(t)}\right|^{p}\right)^{1 / p}}\right)\left(\frac{z_{j}^{(t)}}{\left(\sum_{k=1}^{n}\left|z_{k}^{(t)}\right|^{p}\right)^{1 / p}}\right)\right] \\
& \leqslant \mathbb{E}_{t}\left[\left(\sum_{k=1}^{n}\left|z_{k}^{(t)}\right|^{p}\right)^{2 / p} \Delta^{*}\right] \quad\left(\text { By the definition of } \Delta^{*}\right) \\
& \leqslant\left(\sum_{k=1}^{n} \mathbb{E}_{t}\left[\left|z_{k}^{(t)}\right|^{p}\right]\right)^{2 / p} \Delta^{*} \quad(\text { By Jensen's inequality since } p>2) \\
& \leqslant\left(\sum_{k=1}^{n}\left[\mathbb{E}\left[\left|h_{k}\right|^{p}\right]+1 / n^{4}\right]\right)^{2 / p} \Delta^{*} \quad(\text { By Lemma 6.2) } \\
& \left.=\left(\sum_{k=1}^{n}\left[\gamma_{p}^{p}\left(\mathbb{E}^{*}\left[\left|h_{k}\right|^{2}\right]\right)^{p / 2}+1 / n^{4}\right]\right)^{2 / p} \Delta^{*} \quad \text { (By Defintion of } \gamma_{p}\right) \\
& \leqslant \gamma_{p}^{2}\left(1+1 / n^{3}\right)^{2 / p} \Delta^{*} \quad(\text { By Equation 61) }
\end{aligned}
$$

Since the parameter $n$ is large enough the above analysis proves the approximation achieved by the rounding algorithm. This completes the proof of Theorem 3.7 and concludes this section.

\section{References}

[1] N. Alon, K. Makarychev, Y. Makarychev, and A. Naor. Quadratic forms on graphs. In $37^{\text {th }}$ ACM STOC, pages 486-493, 2005. 4 
[2] N. Alon and A. Naor. Approximating the cut-norm via Grothendieck's inequality. SIAM J. Comput., 35(4):787-803, 2006. 4, 5

[3] S. Arora, B. Barak, and D. Steurer. Subexponential algorithms for Unique Games and related problems. In $51^{\text {st }}$ IEEE FOCS, 2010. 3, 6

[4] S. Arora, E. Berger, E. Hazan, G. Kindler, and M. Safra. On non-approximability for quadratic programs. In $46^{\text {th }}$ IEEE FOCS, pages 206-215, 2005. 5

[5] S. Arora, S. Khot, A. Kolla, D. Steurer, M. Tulsiani, and N. K. Vishnoi. Unique games on expanding constraint graphs are easy: extended abstract. In STOC, pages 21-28, 2008. 3, 6

[6] N. Bansal and S. Khot. Optimal Long Code test with one free bit. In $50^{\text {th }}$ IEEE FOCS, pages 453-462, 2009. 6

[7] N. Bansal and S. Khot. Inapproximability of hypergraph vertex cover and applications to scheduling problems. In ICALP (1), pages 250-261, 2010. 6

[8] I. Bomze. Regalrity versus degeneracy in dynamics, games and optimization: a unified approach to different aspects. SIAM Review, 44(3):394-414, 2002. 6

[9] A. Brieden, P. Gritzman, and V. Klee. Inapproximability of some geometric and quadratic optimization problems. In Approximation and Complexity in Numerical Optimization: Continuous and Discrete Problems, 2000. 5

[10] M. Charikar, K. Makarychev, and Y. Makarychev. Near-optimal algorithms for unique games. In Proc. $38^{\text {th }}$ ACM STOC, pages 205-214, 2006. 6

[11] M. Charikar and A. Wirth. Maximizing quadratic programs: Extending grothendieck's inequality. In $45^{\text {th }}$ IEEE FOCS, pages 54-60, 2004. 4, 5

[12] A. Deshpande, M. Tulsiani, and N. Vishnoi. Algorithms and hardness for subspace approximation. In Proc. SODA, 2011. 1, 3, 5, 7

[13] A. Deshpande and K. R. Varadarajan. Sampling-based dimension reduction for subspace approximation. In STOC, pages 641-650, 2007. 5

[14] N. Devanur, S. Khot, R. Saket, and N. Vishnoi. Integrality gaps for sparsest cut and minimum linear arrangement problems. In Proc. $38^{\text {th }}$ ACM STOC, pages 537-546, 2006. 6

[15] D. Feldman, M. Monemizadeh, C. Sohler, and D. P. Woodruff. Coresets and sketches for high dimensional subspace approximation problems. In SODA, pages 630-649, 2010. 5

[16] V. Feldman, V. Guruswami, P. Raghavendra, and Y. Wu. Agnostic learning of monomials by halfspaces is hard. In Proc. 50 ${ }^{\text {th }}$ IEEE FOCS, 2009. 5, 7, 9

[17] G. Golub and C. van Loan. Matrix Computations. Johns Hopkins University Press, 1996. 5

[18] A. Gupta and K. Talwar. Approximating unique games. In SODA, pages 99-106, 2006. 6

[19] E. Halperin and E. Hazan. HAPLOFREQ-estimating haplotype frequencies efficiently. Journal of Computational Biology, 13(2):481-500, 2006. 6 
[20] S. Har-Peled and K. R. Varadarajan. Projective clustering in high dimensions using core-sets. In Symposium on Computational Geometry, pages 312-318, 2002. 5

[21] S. Khot. Hardness results for coloring 3-colorable 3-uniform hypergraphs. In $43^{\text {rd }}$ IEEE FOCS, pages 23-32, 2002. 7

[22] S. Khot. On the power of unique 2-prover 1-round games. In Proc. $34^{\text {th }}$ ACM STOC, pages 767-775, 2002. 3, 5, 6

[23] S. Khot, G. Kindler, E. Mossel, and R. O'Donnell. Optimal inapproximability results for MAXCUT and other 2-variable CSPs? SIAM J. Comput., 37(1):319-357, 2007. 3, 4, 6

[24] S. Khot and O. Regev. Vertex cover might be hard to approximate to within 2-epsilon. J. Comput. Syst. Sci., 74(3):335-349, 2008. 3, 6

[25] S. Khot and R. Saket. A 3-query non-adaptive PCP with perfect completeness. In Proc. IEEE CCC, pages 159-169, 2006. 7

[26] S. Khot and R. Saket. On hardness of learning intersection of two halfspaces. In Proc. $40^{\text {th }}$ ACM STOC, pages 345-354, 2008. 5, 7

[27] S. Khot and R. Saket. SDP integrality gaps with local $\ell_{1}$-emmbeddability. In Proc. $50^{\text {th }}$ IEEE FOCS, 2009. 6

[28] S. Khot and N. Vishnoi. The unique games conjecture, integrality gap for cut problems and embeddability of negative type metrics into $l_{1}$. In Proc. $46^{\text {th }}$ IEEE FOCS, pages 53-62, 2005. 6

[29] G. Kindler, A. Naor, and G. Schechtman. The UGC hardness threshold of the $l_{p}$ Grothendieck problem. Math. Oper. Res., 35(2):267-283, 2010. 1, 4, 5, 6, 7, 8, 9, 14

[30] A. Kolla. Spectral algorithms for Unique Games. In IEEE Conference on Computational Complexity, pages 122-130, 2010. 6

[31] A. Megretski. Relaxations of quadratic programs in operator theory and system analysis. In Systems, approximation, singular integral operators, and related topics (Bordeaux), number 3, pages 365-392, 2001. 5

[32] E. Mossel. Gaussian bounds for noise correlation of functions and tight analysis of long codes. In $49^{\text {th }}$ IEEE FOCS, pages 156-165, 2008. 4

[33] A. Naor and G. Schechtman. An approximation scheme for quadratic form maximization on convex bodies. Manuscript. 1, 4, 8

[34] A. Nemirovski, C. Roos, and T. Terlaky. On maximization of quadratic form over intersection of ellipsoids with common center. Math. Program., 86(3, Ser. A):463-473, 1999. 4, 5

[35] Y. Nesetrov. Global quadratic optimization via conic relaxation. Working Paper CORE, 1998. 5

[36] P. Raghavendra. Optimal algorithms and inapproximability results for every CSP? In Proc. $40^{\text {th }}$ ACM STOC, pages 245-254, 2008. 3, 6 
[37] P. Raghavendra and D. Steurer. Integrality gaps for strong SDP relaxations of Unique Games. In Proc. $50^{\text {th }}$ IEEE FOCS, 2009. 6

[38] N. D. Shyamalkumar and K. R. Varadarajan. Efficient subspace approximation algorithms. In $S O D A$, pages 532-540, 2007. 5

[39] L. Trevisan. Approximation algorithms for unique games. In $46^{\text {th }}$ IEEE FOCS, pages 197-205, 2005. 6

[40] K. R. Varadarajan, S. Venkatesh, Y. Ye, and J. Zhang. Approximating the radii of point sets. SIAM J. Comput., 36(6):1764-1776, 2007. 5 